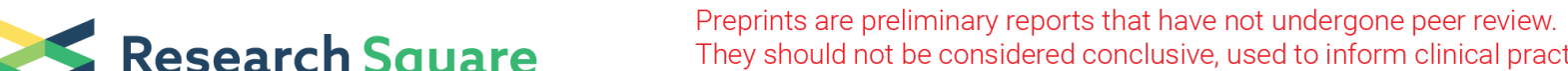 Research Square They should not be considered conclusive, used to inform clinical practice, or referenced by the media as validated information.
}

\section{Evaluating the Prognostic Performance of a Polygenic Risk Score for Breast Cancer Risk Stratification}

Maria Olsen ( $\nabla$ m.olsen@amsterdamumc.nl )

Amsterdam University Medical Centres: Amsterdam Universitair Medische Centra

https://orcid.org/0000-0001-8246-1510

Krista Fischer

University of Tartu Institute of Mathematics and Statistics: Tartu Ulikool Matemaatika ja statistika instituut

Patrick M. Bossuyt

Amsterdam UMC - Locatie AMC: Amsterdam UMC Locatie AMC

Els Goetghebeur

Ghent University: Universiteit Gent

\section{Research article}

Keywords: Prognostic accuracy, Breast cancer, Polygenic risk score, Precision screening, Risk stratification, Medical test evaluation, Biomarker evaluation, Performance measures

Posted Date: December 8th, 2020

DOI: https://doi.org/10.21203/rs.3.rs-121122/v1

License: (c) (1) This work is licensed under a Creative Commons Attribution 4.0 International License. Read Full License

Version of Record: A version of this preprint was published at BMC Cancer on December 1st, 2021. See the published version at https://doi.org/10.1186/s12885-021-08937-8. 


\section{Abstract}

Background: Polygenic risk scores (PRS) could potentially improve breast cancer screening recommendations. We analyzed how well a recently developed prevalence-based breast cancer PRS (Läll et al., 2009) performs in expressing women's future risk of incident breast cancer.

Objectives: To evaluate the prognostic performance of models using PRS and age as predictors, vs age alone, for breast cancer incidence in women from the Estonian biobank (EstBB) cohort.

Methods: We analyzed data on 30,312 women from the EstBB cohort. They entered the cohort between 2002 and 2011, were between 20-89 years, without history of breast cancer, and with full 5-year follow-up by 2015. We examined PRS and other potential risk factors as possible predictors in Cox regression models for breast cancer incidence. With 10 -fold cross validation we estimated 3 - and 5-year breast cancer incidence from age alone and PRS plus age, fitting models on $90 \%$ of the data. Calibration, discrimination, and reclassification then expressed prognostic performance on the left-out folds.

Results: A total of 101 (3.33\%o) and 185 (6.1\%o) incident breast cancers were observed within 3 and 5 years, respectively. For women in the current Estonian screening age (50-62 years), the ratio of observed vs PRS-age modelled 3-year incidence was 0.86 for women in the 75-85\% PRS-group, 1.34 for the $85-95 \%$ PRS-group, and 1.41 for the top 5\% PRS-group. For 5-year incidence, this was respectively $0.94,1.15$, and 1.08. Yet, the number of breast cancer events were relatively low in each PRS-subgroup. The model's AUC was 0.720 (95\% Cl: $0.675-0.765)$ for 3-year and 0.704 (95\% Cl: 0.670-0.737) for 5-year, respectively, just 0.022 and 0.023 higher than for the model with age alone. Using a $1 \%$ risk prediction threshold, the 3-year NRI for the PRS-age model was 0.09 and 0.05 for 5 years.

Conclusion: The model including PRS had modest incremental performance. This suggests that the potential benefit of adding PRS to age for guiding screening likely affects a relatively small proportion of women. A larger, independent study is needed to assess whether and how the PRS can meaningfully contribute to developing more efficient screening strategies.

\section{Introduction}

Breast cancer is the most common female cancer worldwide. In 2018, the age-standardized incidence rate per 100.000 women worldwide was estimated at 46.3, ranging between world regions with 94.2 for Australia and New Zealand to 25.9 for South Central Asia(1), while reaching 113.2 for Belgium as the world's highest rate(2) and a rate of 61.2 for Estonia(3).

Breast cancer screening programs aim to detect tumors before they develop into symptomatic, more advanced cancers, as early detection of localized breast cancer (i.e. cancers that has not yet metastasized) is known to have a better prognosis (4). Current screening recommendations and guidelines rely on a careful weighing of benefits - primarily a reduction in breast cancer specific mortality - 
against adverse events and perceived harms, such as psychological distress due to false positive mammography results and the potential of overdiagnosis(5-8).

Research efforts have produced several discoveries and breakthroughs in breast cancer, from a better understanding of the disease to improved diagnosis, treatment, and management, both in breast cancer patients and in identified high-risk groups(9). So far, this has led to few changes in breast cancer mass screening strategies, where age is still the dominant risk marker for identifying the target group for population screening programs. Today, most health institutes and organizations recommend biennial screening of women from the age of around $40-50$ years to $70-75$ years $(5,10,11)$.

Although the incidence of breast cancer increases significantly with age $(12,13)$, most women do not develop breast cancer. Consequently, a majority of screened women are exposed to the associated harms without reaping the benefits(14). Furthermore, a systematic review reported a cumulative false negative proportion of screenings varying from $8-12 \%$, over a 10-year biennial screening interval of women aged 50-69 years(15). This justifies a quest for additional risk markers to improve the currently implemented breast cancer screening programs. Introducing risk-based screening by targeting those most in need may contribute to the development of more effective programs, with improved benefits or reduced harms, which in turn could lead to improved outcomes of breast cancer screening.

Through GWAS studies more than 100 low penetrance genes, the so-called single nucleotide polymorphisms (SNPs), have been identified as prognostic for breast cancer(16). In contrast to single, rare high-penetrance genes, such as BRCA1/2, these SNPs are single-gene variations that are common in the population and relatively weakly associated with breast cancer, typically at a (GWAS) significance threshold of $5 \times 10^{-8}(17)$. When the effects of SNP variants are combined into a score, the resulting polygenic risk score (PRS) may be used as a risk marker for identifying subgroups that differ in their genetic risk of developing breast cancer $(17,18)$.

Several studies have shown that women with a high PRS are at increased risk of breast cancer(19-26). Läll and colleagues from the Estonian Genome Center recently developed and compared 7 different types of breast cancer PRS, of which one (metaGRS ${ }_{2}$ ) showed a HR of 4.2 (95\% Cl: 2.8-6.2), when women from the Estonian Biobank Cohort (EstBB) in the 95th percentile (the top 5\% PRS group) were compared to women in the lowest 50th percentile PRS group(27).

So far, the validity of the PRS has mostly been demonstrated by reporting relative risks(27). Before we consider involving PRS in screening strategies, we should also evaluate their prognostic performance in expressing risk (incidence), in terms of calibration (relative and absolute agreement between the observed and estimated incidence), discrimination (the ability to correctly classify women who develop breast cancer), and reclassification (the ability to correctly re-classify women who later develop breast cancer into a higher risk group, given a specified risk threshold(28)). Such performance measures could contribute to evidence-based decisions about implementing a PRS in population-based breast cancer screening programs. 
We aimed to estimate 3- and 5-year breast cancer incidence from a Cox-model including either age alone or PRS and age as predictors, for women without current breast cancer and no history of breast cancer in the EstBB cohort. We assessed the prognostic performance of the model including age and PRS in terms of calibration, discrimination, and reclassification.

\section{Methods}

\section{Study cohort}

\section{Recruitment and description}

We included de-identified data from 30,901 female participants from the EstBB cohort. In brief, the EstBB is managed by the Estonian Genome Center at the University of Tartu (EGCUT) and was established to collect genetic and health information, from a large sample of the Estonian population, to advance public health(29-31).

Eligible participants were 18 years or older volunteers with Estonian nationality. Approximately 10.000 participants were recruited from 2002 to 2004. Recruitment was thereafter paused until 2006 due to financial circumstances, but continued again from 2006 to 2012, from all of Estonia (15 counties), at which the EstBB cohort had included almost 52,000 participants.

Participants were initially recruited through general practitioners. Recruitment was later extended to private practices and hospitals, and special recruitment offices of the EGCUT. Recruitment was completely volunteer-based, meaning that no direct contact to the Estonian population was allowed (e.g. through invitation letters) but participants had to actively sign up after hearing about the cohort study in private, at their health care institution, or through promotion at special public events and in the media (29, 30).

By 2012, the number of participants in the EstBB cohort corresponded to approx. $5 \%$ of the adult Estonian population; $66 \%$ were women. The majority is of Estonian ethnicity ( $81.2 \%)$ but the cohort also includes Russian (15.4\%), Ukrainian (1.3\%), and Belarusian (0.6\%) ethnicities. According to the Estonian census report from 2000(32), the EstBB cohort overall represents the general population well although there is an overrepresentation of women (by approx. 10\%), of younger- and middle-age generations, and of people with a higher and professional secondary educational level $(29,30)$.

\section{Eligibility}

Included in this analysis were data from women from the EstBB cohort between 20-89 years and without a current or previous diagnosis of breast cancer at the time of cohort entry. We excluded women who had entered the cohort after 2011, as their 3- and 5-year follow-up information was not yet available at the time of analysis (Fig. 1).

\section{Data collection}


Information on all variables used in this analysis (smoking status, educational level, prevalent comorbidities, age, BMI, and PRS) was initially collected on the day of recruitment through standardized interviews and questionnaires, blood samples, and from existing medical records. Diseases were classified according to the international Classification of Diseases (ICD-10)(29, 30).

The PRS (metaGRS ${ }_{2}$ ) used in this analysis was developed by Läll and colleagues. In brief, they develop several different PRS (GRS), based on the principle that the individual effect of identified breast cancer SNPs, each weighted by their corresponding logistic beta-coefficients (most often from GWAS), can be linearly combined into a single summary PRS-value. A person's individual PRS-value is then the weighted sum of the SNPs that this person carries $(17,27)$.

Läll et. al derived 7 different PRS for the EstBB cohort in three main steps. They first derived two PRS (a $\mathrm{GRS}_{70}$ and $\mathrm{GRS}_{75}$, respectively) based on two different sets of SNPs from other recent PRS publications. They then derived several other PRS based on summary statistics from two recent GWAS studies (the Breast Cancer Association Consortium and the UK Biobank), from which they selected the PRS with the 2 smallest $p$-values for the association with breast cancer ( $\left(\mathrm{GRS}_{\mathrm{ONCO}}\right.$ and $\mathrm{GRS}_{\mathrm{UK}}$ ), using logistic regression analysis on the EstBB cohort (319 women with prevalent breast cancer and 2000 women without prevalent breast cancer). Thereafter, they derived 3 meta-PRS based on (i) the weighted average of the 4 individual PRS (metaGRS ${ }_{4}$ ), (ii) the weighted average of the three strongest associated PRS (metaGRS ${ }_{3}$ ), and (iii) the weighted average of the two strongest associated PRS (metaGRS ${ }_{2}$ ). Of all seven PRS, metaGRS ${ }_{2}$ showed the strongest association with breast cancer and was selected for this analysis. More details about the PRS can be found on the full report and supplementary files of Läll et. al (27).

Follow-up information on incident breast cancers and deaths was collected through biennial linkage to the Estonian Health Insurance Fund, the Estonian Causes of Death Registry, and the Estonian Cancer registry. Every recorded diagnosis of breast cancer was confirmed by an oncologist. The last linkages used in this analysis were performed in December 2015 for breast cancer and in June 2017 for death (27). Below, we studied incident diagnosed breast cancer and death after entry into the EstBB cohort. More information about the EstBB cohort and the PRS can be found elsewhere $(27,29-31,33)$.

\section{Statistical analyses}

Descriptive statistics for our study group are presented in Table 1. For continuous covariates (age, BMI, and follow-up time) we show the mean and median as well as standard deviation (SD) and interquartile range (IQR), and the number (n) and proportion (\%) for the categorical covariates (education, smoking, prevalent co-morbidities).

PRS results were categorized into 6 subgroups $(0-25 \%, 25-50 \%, 50-75 \%, 75-85 \%, 85-95 \%$ and the top $5 \%$ PRS percentiles) in accordance with the analysis performed by Läll et al (27). We subsequently fitted a full multivariable Cox proportional hazard regression model with main effects of age, BMI, year of entry, 6-level categorical predictors of PRS, smoking status (never/former/current), education (less than 
secondary/secondary/university degree), and prevalent co-morbidities (any prevalent cancer, Type 1 diabetes, Type 2 diabetes, myocardial infarction, and coronary artery disease), together with interaction terms for BMI and age, and PRS and age. Follow-up time was measured in years from cohort entry to last linkage.

Only two covariates were statistically significant predictors and retained in subsequent models: age and PRS. We then fitted two Cox regression models: one including only age, to reflect the current screening strategy, the second adding PRS to age as predictor. From the latter PRS-age-based model fitted to the full data set, we showed breast cancer specific hazard ratios with main effects for age and PRS-groups (Table 2).

The proportionality assumption for both PRS and age was formally tested using Schoenfeld's residuals (Supplementary Figure S1) and the fitted PRS-age-model and age-model were compared with a likelihood ratio test. We estimated cause specific hazards of 'death without breast cancer' and 'breast cancer' from Cox regressions for each woman for 3 and 5 years, and evaluated the cumulative incidence of breast cancer from both the PRS-age model and age-model. Below, we referred to these model-estimated cumulative incidences as PRSage_mlnc and age_mlnc, respectively. They express the calculated probability of developing a clinically diagnosed breast cancer within the corresponding follow-up period. We analyzed and compared the distributions of age_mlnc and PRSage_mlnc in the study group.

To avoid overoptimistic incidence estimates from the fitted models, we evaluated each model's performance using 10 -fold cross-validation. We randomly split the data into 10 equally sized parts, of which 9 parts were used to fit the model and derived 3-and 5-year estimated incidences for the remaining 10th part test set. This process was repeated 10 times, once for each distinct left-out test set. All 10 testsets were then combined into the merged data set with their independently estimated incidences. This was used to evaluate risk distribution, calibration, discrimination, and reclassification for both PRSage_mlnc and age_mlnc.

Unadjusted cumulative incidence curves over time since study entry until last follow-up of breast cancer (in years) were constructed separately for observed breast cancer and death without breast cancer, stratified by PRS-groups (Fig. 2).

Since the current Estonian screening program invites women from the age of 50 to 62 years for biennial screening(34), we constructed three age-groups of women: $<50$ years, 50 to 62 years, and $>62$ years. For evaluating calibration, we combined the PRS and age-groups, which resulted in 18 different PRS-agesubgroups.

Our calibration plots show for each PRS-age-subgroup the observed cumulative incidence against the mean PRSage_mlnc for the same group. The observed cumulative incidence was calculated as the proportion (\%) of breast cancer events within 3 or 5 years among the total number of women in each PRS-age-subgroup, with corresponding $95 \% \mathrm{Cl}$ calculated using the Wilson method (Fig. 4, Table 3). Per study design, no censoring was encountered over the periods envisaged. 
Receiver operating characteristic (ROC) curves were constructed and the corresponding area under the curves (AUC) was calculated for the age_mInc and PRSage_mlnc, for both the 3-and the 5-year time points. The $95 \% \mathrm{Cl}$ of the AUC was calculated with a non-parametric method (Delong) (Fig. 5).

Using a risk threshold of $\geq 1 \%$, we classified women as high or low risk. We constructed reclassification tables and calculated the net-reclassification index (NRI) to explore how the PRSage_mInc reclassified women into high- and low-risk groups, compared to classification by the age_mlnc. Hence, within the group of women with breast cancer the absolute number and proportions of correctly reclassified women (i.e. women moving from an age_mlnc below $1 \%$ to a PRSage_mlnc $\geq 1 \%$ ) and incorrectly reclassified women (i.e. women moving from an age_mlnc $\geq 1 \%$ to an PRSage_mlnc below 1\%) were calculated. This was likewise calculated for the group of women without breast cancer, but with the opposite direction for correct reclassifications (i.e. women moving from an age_mlnc $\geq 1 \%$ to a PRSage-mlnc below $1 \%$ ) and incorrect reclassifications (i.e. women moving from an age_mlnc below $1 \%$ to a PRSage_mlnc $\geq 1 \%$ ). As an overall summary measure, the NRI was then calculated by first subtracting the incorrect reclassifications from the current ones in each group, and adding the two proportions. The $95 \% \mathrm{Cl}$ intervals of the NRI were calculated as proposed by Pencina et al, 2008 (35) (Tables 5 and 6).

P-values below 0.05 were considered to indicate statistically significant differences. All statistical analyses were performed using RStudio (Version 1.1.463, 2009-2018 RStudio, Inc.)

\section{Results}

Of the 30,901 women from the EstBB cohort between 20-89 years, 309 women had prevalent breast cancer at recruitment while 227 women had been recruited after 2011. Hence, a total of 30,365 women were eligible, of which 30,312 had complete data and were included in the analyses (Fig. 1). Demographic and clinical characteristics of included women are presented in Table 1, and per PRS-groups and agegroups in Supplementary Table S1, S2, and Figure S2. 
Table 1

Demographic and clinical characteristics of included participants

\begin{tabular}{|c|c|c|}
\hline \multirow[t]{2}{*}{ Characteristic } & & $\mathrm{n}(\%)$ \\
\hline & & mean / median (SD; IQR) \\
\hline \multicolumn{2}{|l|}{ Total included } & 30,312 \\
\hline \multicolumn{2}{|l|}{ Follow-up time (by June 2017) } & 9 / 8.59 years $(2.6 ; 7.48-9.47)$ \\
\hline \multicolumn{2}{|l|}{ Age } & $46 / 45$ years $(17 ; 32-58)$ \\
\hline \multicolumn{2}{|l|}{ BMI } & 26.44 / $25.39(5.69 ; 22.21-29.74)$ \\
\hline \multirow[t]{3}{*}{ Smokers } & Currently: & $6,884(23 \%)$ \\
\hline & Former: & $3,087(10 \%)$ \\
\hline & Never: & $20,341(67 \%)$ \\
\hline \multirow[t]{3}{*}{ Education } & Less than secondary: & $4,077(14 \%)$ \\
\hline & Secondary: & $17,979(59 \%)$ \\
\hline & University degree: & $8,256(27 \%)$ \\
\hline \multirow[t]{5}{*}{ Co-morbidities } & Mean number: & 0.2336 (range: $0-5$ ) \\
\hline & With 0: & $24,962(82 \%)$ \\
\hline & with 1: & $3,952(13 \%)$ \\
\hline & with 2: & $1117(4 \%)$ \\
\hline & With 3-5: & $281(1 \%)$ \\
\hline
\end{tabular}

\section{Observed and unadjusted cumulative incidence}

The observed cumulative incidence of breast cancer in the entire cohort was 3.33\%o (101 events) at 3 years and $6.0 \%$ o (185 events) at 5 years, while it was twice as high in the top 5\% PRS-group, at both 3 (6.6\%o) and 5 years (13.19\%o) (Supplementary Table S9 and S10).

Unadjusted cumulative breast cancer incidence increased with higher PRS scores, while there was no such PRS based difference was observed for the unadjusted cumulative incidence of death (Fig. 2).

\section{Proportional hazard models}

The PRS-age-based model fitted the data significantly better than the age-based model ( $p$-value $=1.75 e-$ 14) with no significant improvement in model fit for the full model, with all evaluated putative predictors 
$(p-v a l u e=0.25)$.

The estimated age-adjusted effect of the PRS on the breast cancer specific hazard increased with the rank order of the PRS-groups, with HR ranging from 1.2 (95\% Cl: $0.81-1.84)$ to 4.6 (95\% Cl: 2.97-7.14), relative to the lowest $0-25 \%$ PRS subgroup (Table 2).

Table 2

Breast cancer specific hazard ratios with main effects for age and PRS-groups

\begin{tabular}{|llll|}
\hline Variable & $\begin{array}{l}\text { Age-adjusted HR } \\
\text { exp(coef) }\end{array}$ & $\begin{array}{l}\text { Lower } \\
\mathbf{9 5 \%} \mathrm{Cl}\end{array}$ & $\begin{array}{l}\text { Upper } \\
\mathbf{9 5 \%} \mathrm{Cl}\end{array}$ \\
\hline Age (standardized)* & 1.83 & 1.63 & 2.05 \\
\hline $0-25 \%$ & Reference & - & - \\
\hline $25-50 \%$ & 1.22 & 0.81 & 1.84 \\
\hline $50-75 \%$ & 1.88 & 1.30 & 2.7 \\
\hline $75-85 \%$ & 2.32 & 1.50 & 3.58 \\
\hline $85-95 \%$ & 3.45 & 2.31 & 5.13 \\
\hline Top 5\% & 4.60 & 2.97 & 7.14 \\
\hline * SD of age is 17 & & & \\
\hline
\end{tabular}

\section{Risk distributions}

From the Cox models, we estimated the 3- and 5-year breast cancer specific cumulative incidence for women in the cohort with the age-based model (age_mlnc) and with the age and PRS-based model PRSage_mlnc).

The PRSage_mlnc distribution showed a slightly wider spread than age_mlnc, best visible in the righthand tail for both the 3- and 5-year estimated incidence (Fig. 3 and Supplementary Table S3). For example, 228 women had a 3-year age_mlnc »1\% compared to 1,115 women for PRSage_mlnc: a difference of 887 women (3\% of the cohort). No woman had a 3-year age_mlnc of $1.5 \%$ or greater while 322 women had so with the PRSage_mlnc (Supplementary Table S4-S5).

For the 5-year risk distributions, 4,679 women had an age_mlnc of $1 \%$ or greater versus 4,644 women with the PRSage_mlnc (difference of $0.1 \%$ for the entire cohort). For the $1.5 \%$ risk threshold, the numbers were 833 versus 1848 women, for the age_mlnc and PRSage_mlnc, respectively (Supplementary Table S6-S7).

The ability of the PRS to stratify women into different risk categories was furthermore demonstrated by an, although modest, variation of the PRSage_mlnc with age. The average age-based risk (non-cross 
validated) of women who enter the Estonian screening program at 50 years was reached at different ages in each of the PRS groups. Particularly in the $85-95 \%$ and top 5\% PRS-groups, this average risk was reached at a much younger age than 50 years (Supplementary Figure S3).

\section{Performance}

We evaluated the prognostic performance of PRSage_mInc in terms of calibration, discrimination, and reclassification, comparing it to age_mlnc whenever appropriate.

\section{Calibration}

95\% Cls for the observed incidences were wide: 1.55 to $7.35 \%$ ond 3.39 to $11.04 \%$ o at 3 and 5 years, respectively. Calibration varied across the PRS-age subgroups, however, the estimated incidences were still within the $95 \% \mathrm{Cl}$ for all subgroups (Fig. 4). More details on calibration for the 3-year observed incidence and PRSage_mlnc across all PRSage subgroups are shown in Table 4 and for the 5-year incidences in Supplementary Table S8.

For women in the current screening age (50-62 years), the ratio of observed vs PRSage_mlnc was 0.86 for women in the $75-85 \%$ PRS-group, 1.34 for the $85-95 \%$ PRS-group, and 1.41 for the top $5 \%$ PRSgroup. For 5-year PRSage_mlnc, these were $0.94,1.15$, and 1.08, respectively.

The approximation of PRSage_mlnc to observed incidence was similar for the top 3 PRS group for women below 50 years and also for the age group above 62 years, with exemption of the groups of women in the top 5\% PRS-group and older than 62 years $(n=265)$, for which the PRSage_mlnc was considerably overestimated. Yet, the number of breast cancer events were low in each of the PRS-age subgroups.

When calibration was assessed for all women in the top 5\% PRS-group $(n=1516)$ we also observed an overestimation, but limited to a ratio of 0.80 for 3-year and 0.88 for 5-year PRSage_mlnc (Supplementary Table S9-S10). An almost perfect calibration was observed for the larger subgroup of the highest 75$100 \%$ PRS $(n=7569)$ with respective ratios of 0.97 and 1.00 , while an underestimation was observed for the lowest $0-25 \%$ PRS-group $(n=7584)$, with a ratio 1.24 for 3-year and 1.20 for 5-year PRSage_mlnc (Supplementary Table S9-S10). 
Table 3

Three-year observed cumulative incidence and PRSage_mlnc per 1000 women

\begin{tabular}{|c|c|c|c|c|c|c|}
\hline $\begin{array}{l}\text { PRS-age- } \\
\text { group }\end{array}$ & $\begin{array}{l}\text { Number } \\
\text { of } \\
\text { women } \\
\text { (n) }\end{array}$ & $\begin{array}{l}\text { Number of } \\
\text { breast cancers } \\
\text { in } 3 \text { years (n) }\end{array}$ & $\begin{array}{l}\text { 3-year } \\
\text { observed } \\
\text { Incidence (95\% } \\
\mathrm{Cl})^{*}\end{array}$ & $\begin{array}{l}\text { 3-year mean } \\
\text { PRSage_mlnc }\end{array}$ & Difference & Ratio \\
\hline $\begin{array}{l}0-25 \% \\
\text { PRS; } 18- \\
49 \text { years }\end{array}$ & 4499 & 10 & $\begin{array}{l}2.22(1.21- \\
4.09)\end{array}$ & 1.06 & 1.16 & 2.09 \\
\hline $\begin{array}{l}25-50 \% \\
\text { PRS; } 18- \\
49 \text { years }\end{array}$ & 4466 & 2 & $\begin{array}{l}0.45(0.12- \\
1.63)\end{array}$ & 1.30 & -0.85 & 0.35 \\
\hline $\begin{array}{l}50-75 \% \\
\text { PRS; } 18- \\
49 \text { years }\end{array}$ & 4494 & 7 & $\begin{array}{l}1.56(0.75- \\
3.21)\end{array}$ & 2.01 & -0.45 & 0.78 \\
\hline $\begin{array}{l}75-85 \% \\
\text { PRS; } 18- \\
49 \text { years }\end{array}$ & 1794 & 6 & $\begin{array}{l}3.34(1.53- \\
7.28)\end{array}$ & 2.46 & 0.88 & 1.36 \\
\hline $\begin{array}{l}85-95 \% \\
\text { PRS; } 18- \\
49 \text { years }\end{array}$ & 1789 & 5 & $\begin{array}{l}2.79(1.19- \\
6.53)\end{array}$ & 3.67 & -0.87 & 0.76 \\
\hline $\begin{array}{l}\text { Top 5\% } \\
\text { PRS; } 18- \\
49 \text { years }\end{array}$ & 895 & 4 & $\begin{array}{l}4.47(1.74- \\
11.43)\end{array}$ & 4.87 & -0.4 & 0.92 \\
\hline $\begin{array}{l}\text { Sub-total; } \\
18- \\
49 \text { years }\end{array}$ & 17937 & 34 & $\begin{array}{l}1.90(0.88- \\
4.19)\end{array}$ & 1.95 & -0.05 & 0.97 \\
\hline $\begin{array}{l}0-25 \% \\
\text { PRS; } 50- \\
62 \text { years }\end{array}$ & 1750 & 1 & $\begin{array}{l}0.57(0.03- \\
3.23)\end{array}$ & 2.20 & -1.63 & 0.26 \\
\hline $\begin{array}{l}25-50 \% \\
\text { PRS; } 50- \\
62 \text { years }\end{array}$ & 1701 & 7 & $\begin{array}{l}4.12(1.99- \\
8.47)\end{array}$ & 2.67 & 1.44 & 1.54 \\
\hline $\begin{array}{l}50-75 \% \\
\text { PRS; } 50- \\
62 \text { years }\end{array}$ & 1704 & 10 & $\begin{array}{l}5.87(3.19- \\
10.77)\end{array}$ & 4.17 & 1.7 & 1.41 \\
\hline $\begin{array}{l}75-85 \% \\
\text { PRS; } 50- \\
62 \text { years }\end{array}$ & 683 & 3 & $\begin{array}{l}4.39(1.49- \\
12.83)\end{array}$ & 5.09 & -0.7 & 0.86 \\
\hline
\end{tabular}

Legend: The table shows the 3-year observed cumulative incidence and mean PRSage_mlnc, for each PRSage subgroup, per 1000 women. The difference is observed incidence minus the mean PRSage_mlnc. The ratio expresses observed incidence over mean PRSage_mlnc. *Confidence intervals were calculated with the Wilson method. 


\begin{tabular}{|c|c|c|c|c|c|c|}
\hline $\begin{array}{l}\text { PRS-age- } \\
\text { group }\end{array}$ & $\begin{array}{l}\text { Number } \\
\text { of } \\
\text { women } \\
\text { (n) }\end{array}$ & $\begin{array}{l}\text { Number of } \\
\text { breast cancers } \\
\text { in } 3 \text { years }(n)\end{array}$ & $\begin{array}{l}\text { 3-year } \\
\text { observed } \\
\text { Incidence }(95 \% \\
\text { Cl)* }\end{array}$ & $\begin{array}{l}\text { 3-year mean } \\
\text { PRSage_mlnc }\end{array}$ & Difference & Ratio \\
\hline $\begin{array}{l}85-95 \% \\
\text { PRS; } 50- \\
62 \text { years }\end{array}$ & 697 & 7 & $\begin{array}{l}10.04(4.87- \\
20.58)\end{array}$ & 7.51 & 2.53 & 1.34 \\
\hline $\begin{array}{l}\text { Top } 5 \% \\
\text { PRS; } 50- \\
62 \text { years }\end{array}$ & 356 & 5 & $\begin{array}{l}14.04(6.01- \\
32.45)\end{array}$ & 9.96 & 4.08 & 1.41 \\
\hline $\begin{array}{l}\text { Sub-total; } \\
50- \\
62 \text { years }\end{array}$ & 6891 & 33 & $\begin{array}{l}4.79(2.24- \\
10.60)\end{array}$ & 4.03 & 0.76 & 1.19 \\
\hline $\begin{array}{l}0-25 \% \\
\text { PRS; } 63- \\
89 \text { years }\end{array}$ & 1335 & 6 & $\begin{array}{l}4.49(2.06- \\
9.77)\end{array}$ & 3.83 & 0.66 & 1.17 \\
\hline $\begin{array}{l}25-50 \% \\
\text { PRS; } 63- \\
89 \text { years }\end{array}$ & 1417 & 8 & $\begin{array}{l}5.65(2.86- \\
11.1)\end{array}$ & 4.70 & 0.95 & 1.2 \\
\hline $\begin{array}{l}50-75 \% \\
\text { PRS; } 63- \\
89 \text { years }\end{array}$ & 1377 & 7 & $\begin{array}{l}5.08(2.46- \\
10.46)\end{array}$ & 7.18 & -2.1 & 0.71 \\
\hline $\begin{array}{l}75-85 \% \\
\text { PRS; } 63- \\
89 \text { years }\end{array}$ & 545 & 4 & $\begin{array}{l}7.34(2.86- \\
18.72)\end{array}$ & 8.67 & -1.33 & 0.85 \\
\hline $\begin{array}{l}85-95 \% \\
\text { PRS; } 63- \\
89 \text { years }\end{array}$ & 545 & 8 & $\begin{array}{l}14.68 \text { (7.46- } \\
28.7)\end{array}$ & 13.17 & 1.51 & 1.11 \\
\hline $\begin{array}{l}\text { Top 5\% } \\
\text { PRS; 63- } \\
89 \text { years }\end{array}$ & 265 & 1 & $\begin{array}{l}3.77(0.19- \\
21.06)\end{array}$ & 17.46 & -13.69 & 0.22 \\
\hline $\begin{array}{l}\text { Sub-total; } \\
63- \\
89 \text { years }\end{array}$ & 5484 & 34 & $\begin{array}{l}6.20(2.89- \\
13.06)\end{array}$ & 6.96 & -0.76 & 0.89 \\
\hline $\begin{array}{l}\text { Total } \\
\text { (entire } \\
\text { cohort) }\end{array}$ & 30312 & 101 & $\begin{array}{l}3.33(1.55- \\
7.35)\end{array}$ & 3.33 & 0.00 & 1.00 \\
\hline \multicolumn{7}{|c|}{$\begin{array}{l}\text { Legend: The table shows the } 3-y e a r \text { observed cumulative incidence and mean PRSage_mlnc, for each } \\
\text { PRSage subgroup, per } 1000 \text { women. The difference is observed incidence minus the mean } \\
\text { PRSage_mlnc. The ratio expresses observed incidence over mean PRSage_mlnc. *Confidence } \\
\text { intervals were calculated with the Wilson method. }\end{array}$} \\
\hline
\end{tabular}

\section{Discrimination}


The ability of the PRSage_mlnc risk to accurately classify women with breast cancer into a high-risk category and women without breast cancer into a low-risk category was assessed by building ROC curves and estimating the corresponding AUC. The PRSage_mlnc model had an AUC of 0.720 (95\% Cl: 0.675 to 0.765 ) for 3-years and 0.704 ( $95 \% \mathrm{Cl}: 0.670$ to 0.737 ) for 5 -years, respectively just 0.022 and 0.023 higher than for the age_mlnc model. Neither increment was statistically significant ( $p$-value $=0.23$ for 3-years and $p$-value $=0.11$ for 5-years, calculated with the Delong test) (Fig. 5).

\section{Reclassification}

We constructed risk classification tables for the age_mlnc and the PRSage_mlnc, using risk threshold of $1 \%$ for both 3- and 5-years (Tables 5 and 6), and scrutinized reclassification through the use of PRSage_mlnc rather than age_mlnc.

The 3-year risk reclassification showed that $13 \%$ of women with breast cancer $(13 / 101)$ were correctly reclassified into a high risk-group while $1 \%(1 / 101)$ of women were wrongly re-classified by the PRSage_mlnc. This resulted in a net-improvement of $12 \%$ correctly re-classified women with breast cancer (Table 5). Among women without breast cancer $(30,211)$, the PRSage_mlnc wrongly re-classified more women as high risk (3\%) than correctly as low risk $(0.4 \%)$, resulting in a 'net-loss' $2.6 \%$ of wrongly upward reclassifications (Table 5). The net reclassification index (NRI) was 0.09 (95\% Cl: 0.02-0.16, p = $0.01)$.

For the 5-year risk, the PRSage_mlnc correctly re-classified $15 \%$ of women with breast cancer as high-risk $(27 / 185)$ but also wrongly classified $10 \%(18 / 185)$ downwards, leading to a net improvement of $5 \%$ correctly reclassifications of women with breast cancer (Table 6). In women without breast cancer $(30,127), 6.7 \%$ were wrongly re-classified upwards, while $6.9 \%$ were correctly re-classified downwards (Table 6), resulting in a net-improvement of $0.2 \%$ and a NRI of $0.05(95 \% \mathrm{Cl}:-0.02-0.12, \mathrm{p}=0.17)$.

Table 5

Reclassification tables of women with 1\% 3-year risk threshold

\begin{tabular}{|lllll|}
\hline $\begin{array}{l}\text { Women with breast } \\
\text { cancer } \\
\text { in } 3 \text { years }\end{array}$ & $\begin{array}{l}\text { PRSage_mlnc } \\
\leq 1 \%\end{array}$ & $\begin{array}{l}\text { PRSage_mlnc } \\
\mathbf{1} \%\end{array}$ & $\mathbf{N}$ & $\begin{array}{l}\text { Re-classified with } \\
\text { PRSage_mlnc (\%) }\end{array}$ \\
\hline age_mlnc $\leq 1 \%$ & 86 & 13 & 99 & $13 / 101=0.13(13 \%)$ \\
\hline age_mlnc $\geq 1 \%$ & 1 & 1 & 2 & $1 / 101=0.01(1 \%)$ \\
\hline N & 87 & 14 & 101 & $0.13-0.01=0.12(12 \%)$ \\
\hline
\end{tabular}




\begin{tabular}{|lllll|}
\hline $\begin{array}{l}\text { Women without breast } \\
\text { cancer } \\
\text { in 3 years }\end{array}$ & $\begin{array}{l}\text { PRSage_mlnc } \\
\leq 1 \%\end{array}$ & $\begin{array}{l}\text { PRSage_mlnc } \\
\geq 1 \%\end{array}$ & $\mathbf{N}$ & $\begin{array}{l}\text { Re-classified with } \\
\text { PRSage_mlnc (\%) }\end{array}$ \\
\hline age_mlnc $\leq 1 \%$ & 28991 & 994 & 29985 & $994 / 30211=0.03(3 \%)$ \\
\hline age_mlnc $\geq 1 \%$ & 119 & 107 & 226 & $119 / 30211=0.004(0.4 \%)$ \\
\hline N & 29110 & 1101 & 30211 & $0.03-0.004=0.026(2.6 \%)$ \\
\hline
\end{tabular}

Table 6

Reclassifications in women with 1\% 5-year risk threshold

\begin{tabular}{|lllll|}
\hline $\begin{array}{l}\text { Women with breast } \\
\text { cancer } \\
\text { in } 5 \text { years }\end{array}$ & $\begin{array}{l}\text { PRSage_mlnc } \\
\leq 1 \%\end{array}$ & $\begin{array}{l}\text { PRSage_mlnc } \\
\mathbf{2 1 \%}\end{array}$ & $\mathbf{N}$ & $\begin{array}{l}\text { Re-classified with } \\
\text { PRSage_mlnc (\%) }\end{array}$ \\
\hline age_mlnc $\leq 1 \%$ & 101 & $\mathbf{2 7}$ & 128 & $27 / 185=0.15(15 \%)$ \\
\hline age_mlnc $\geq 1 \%$ & $\mathbf{1 8}$ & 39 & 57 & $18 / 185=0.1(10 \%)$ \\
\hline N & 119 & 66 & 185 & $0.15-0.1=0.05(5 \%)$ \\
\hline
\end{tabular}

\begin{tabular}{|lllll|}
\hline $\begin{array}{l}\text { Women without breast } \\
\text { cancer } \\
\text { in 5 years }\end{array}$ & $\begin{array}{l}\text { PRSage_mlnc } \\
\leq 1 \%\end{array}$ & $\begin{array}{l}\text { PRSage_mlnc } \\
\mathbf{1} \%\end{array}$ & $\mathbf{N}$ & $\begin{array}{l}\text { Re-classified with } \\
\text { PRSage_mlnc (\%) }\end{array}$ \\
\hline age_mlnc $\leq 1 \%$ & 23473 & 2032 & 25505 & $2032 / 30127=0.067(6.7 \%)$ \\
\hline age_mlnc $\geq 1 \%$ & 2076 & 2546 & 4622 & $2076 / 30127=0.069(6.9 \%)$ \\
\hline N & 25549 & 4578 & 30127 & $\begin{array}{l}0.067-0.069=-0.002 \\
(-0.2 \%)\end{array}$ \\
\hline
\end{tabular}

\section{Discussion}

Although age is a strong risk factor for breast cancer among the general population, additional risk markers may support a more fine-grained risk stratification of women, allowing to improve current screening practices. We evaluated prognostic performance of 3- and 5-year breast cancer incidence in the EstBB cohort as estimated from a Cox proportional hazards model including PRS and age as predictors versus age alone. This happened in terms of calibration, discrimination, and reclassification. Adding PRS to age significantly improved model goodness-of-fit. The 3-year and 5-year PRSage_mlnc were well calibrated. Overall improvements in terms of discrimination and reclassification were, however, modest. 
The large number of participants in the EstBB cohort, together with the sequencing data that were available for each participant and the direct linkage to several Estonian population registries $(29,30,36)$, are key strengths of this study. Even though events per category were relatively limited, we estimated absolute 3- and 5-year incidence from these data which were all uncensored up to 5 years of follow-up. To our knowledge, no other study has examined absolute risk estimates with similar design. Instead, other PRS evaluations have relied on input from external sources to convert relative to absolute risks (21$26,37,38)$.

A number of limitations to our study should also be acknowledged. The EstBB participants were nonrandomly recruited, primarily through general practitioners and other medical sites. Consequently, the included women are younger, slightly more resourceful and better educated than the general Estonian population $(29,30)$. This could limit generalizability of our results. As breast cancer incidence is higher in the middle to older generation, the incidence in our cohort may be underrepresented. However, the observed incidence of breast cancer in our analysis is an accordance with Estonian breast cancer statistics(3).

Though no statistically significant interaction between age and the PRS was observed, we cannot rule out that the PRS may have a stronger discriminating effect on breast cancer risk in younger than in older women. The number of breast cancer events in the EstBB cohort may have been too small for the formal test to have sufficient power. Such a PRS-age interaction has been reported in the PRS evaluation by Mavaddat et al., 2015 and 2019(16, 23).

Lastly, 3- and 5-years are relatively short time horizons for evaluating model-estimated breast cancer incidences, in particular because relatively few incident events have emerged in the cohort. This led to large uncertainty intervals for the top 5\% PRS groups primarily. Yet we believe that these time points are particularly relevant in the evaluation of the PRS for potential use in current practice. As the Estonia screening program invites women for screening every second year and the EstBB cohort had full followup until 5 years, the cohort entry could mimic a 'first screen' in our sample. Indeed, no women in our sample had known current or previous breast cancer and the 3- and 5-year time points roughly correspond to the timings of the two screening rounds following cohort entry.

\section{Interpretation of results}

In agreement with results from Läll et. all and several other PRS evaluations(19-21, 23-25, 27), the PRS was found to be moderately to strongly associated with breast cancer, primarily when comparing the top 3 PRS \% groups to the lowest 0-25\% PRS group. The proportion women who were assigned a substantially higher risk with the PRSage_mlnc was, however, limited to a maximum of $3 \%$ within 3 years and $3.5 \%$ within 5 years.

A PRS developed for prevalent cases can be expected to be less performant for future incidence. Yet in a screening context, the PRS would target such incidence as women with a history of cancer are following other monitoring programs and are, correspondingly, not included in screening of the general population. 
In terms of calibration, the PRSage_mlnc approximated the observed incidence relatively well for each PRS-age-subgroup, as only one exception, of the top 5\% PRS in women older than 62 years, was considerably overestimated for both time points. This overestimation may be owning to the low sample size of this subgroup. Some under- and overestimation is expected at both tails of the PRSage_mlnc. The calibration was more accurate for the highest 75-100\% PRS-group than for the lowest 0-25\% PRSgroup. This too could be explained by the considerably higher observed incidence in the highest $75-100 \%$ PRS-group, with similar total sample size.

The AUC estimates the percentage of (randomly selected) pairs of women with and without breast cancer where the woman with breast cancer has the higher risk prediction, here age_mlnc and PRSage-mInc. It is well-known that extremely high odds ratios or relative risks of new potential risk factors are needed to achieve sizeable improvements in discriminative performance (i.e. increase in AUC)(39-41), and in particular when added to existing risk factors(42). Upon adding the PRS to age, the increase in AUC was limited to $2-3 \%$ more pairs assigning higher risk to the breast cancer case. Although the AUC in our analysis are slightly higher than in other PRS evaluations $(20,21,23-25,37)$, two studies have reported similarly small improvements in AUC of 3-4\% $(21,25)$, while two other studies reported an increase of 7$9 \%$ when adding SNPs/PRS to existing risk factors $(23,24)$.

The AUC do not inform on whether the small improvement could translate into sufficient discrimination of clinically defined subgroups, supporting different screening recommendations. Net-reclassification gives a more direct indication of the incremental performance upon defining potentially clinically relevant riskgroups using a threshold value. With a threshold of $1 \%$ in the reclassification we exemplified defining new PRSage-based risk groups with corresponding screening recommendations.

The 3-year PRSage_minc showed a modest but statistically significant improvement in overall reclassification over the age_mlnc, whereas the improvement was smaller and non-significant for the 5year incidence. The reclassification findings concur with two studies that reported NRI values in a range of approx. $0.08-0.010$ for 5-year incidence $(25,37)$. Several other studies also showed that incremental improvements by SNPS/PRS were primarily restricted to women with breast cancer, while improvements for women without breast cancer was almost absent(24-26, 37). Except for one smaller study, which found that women without breast cancer also shifted downwards(21), these studies overall suggest that the benefits are primarily limited to women with breast cancer, often at a small cost for women without breast cancer.

\section{Implications of our results}

As the general population incidence of breast cancer is relatively low, unnecessary screening of a large group of women is one of the main challenges in breast cancer screening. Association measures such as the HR inform us about the relative differences in breast cancer risk between the PRS groups, but even high relative hazards may not necessarily mean that the PRS estimates the women's future risk of developing breast cancer well. To improve on current age-based screening, the PRS should ideally identify 
a sufficient number of women who are truly at higher risk or, alternatively, avoid unnecessary screenings in a sufficient number of women at truly low risk.

Notwithstanding good calibration, the small improvements in risk stratification and discrimination by the PRS did not translate into a considerable number of additional women correctly reclassified as high- or low-risk. Although the small but positive NRI could be interpreted as a benefit for women with breast cancer, at very little cost of women without breast cancer, the overall benefits for the entire cohort are limited. In summary, this suggest that the potential for the PRS to have sufficiently large implications for screening programs may be limited.

If and to what extent the PRS could meaningfully contribute to the development of more efficient screening strategies could not be fully answered by our data and should be further evaluated. We recognize that this is not a straightforward question, as it also depends on many other factors, such as the screening interval in an alternative strategy, the number of interval cancers, and the outcomes that are evaluated.

\section{Future research}

The wave of incoming data from 150.000 new participants in the EstBB cohort (43-45) will enable a more refined and a more precise evaluation of the estimated incidences in the PRS-age-subgroups. Future evaluations may consider different risk thresholds, further optimize the Cox regression models, but could also use microsimulation models to explore how risk-based screening could bring benefits for the highrisk group in terms of breast cancer specific- and overall mortality. It may also document corresponding potential harms. One could study if women with lower PRS-age-based risk would benefit from larger screening intervals or from postponing screening until they reach the risk of an average 50-year-old. The PRS could also be useful for other applications than mass screening, such as improvements in risk prediction models already implemented in clinical care, e.g. the Gail model.

For complete biomarker evaluation, a large, pragmatic randomized trial would bring valid and convincing evidence of clinical effectiveness. Though few such studies are conducted in biomarker research, several larger intervention trials for PRS-based breast cancer screening have recently been initiated. One is the WISDOM study in US, a prospective randomized trial (Clinical Trials identifier NCT02620852). Another is the My Personalized Breast cancer Screening project (MyPeBS), a large European international initiative(46). A feasibility trial using the PRS that we evaluated has been initiated in Estonia (Clinical Trials identifier NCT03989258).

\section{Conclusion}

Our analysis of women from the EstBB showed low observed cumulative incidence, which varied across PRS percentile groups. Adding the PRS significantly improved the fit of Cox proportional hazard model based on age only. The 3- and 5-year PRSage_mlnc approximated the observed incidence for each PRSage group relatively well. Through cross validation, PRS-age-based incidence showed modest 
incremental prognostic performance on the test-data, compared to age-based incidence, in terms of discriminatory ability and reclassification. Further research is needed to assess whether and how PRS can contribute to more effective breast cancer screening strategies.

\section{Abbreviations}

age_mlnc: Age-modelled incidence (age_mlnc). Absolute cross-validated model-based breast cancer incidences, estimated with a Cox Proportional regression model including only age as covariate. $t$ is the time window, of 3- and 5-years, over which the incidence was estimated; AUC: Area under the curve; $\mathrm{Cl}$ : Confidence intervals; EGCUT: Estonian Genome Center at the University of Tartu; EstBB: Estonian Biobank; HR: Hazard ratio; IQR: Interquartile range; NRI: Net reclassification index; PRSage_mlnc: PRSage-modelled incidence (PRSage_mlnc). Absolute cross-validated model-based breast cancer incidences, estimated with a Cox Proportional regression model including PRS plus age as covariates. $t$ is the time window, of 3- and 5-years, over which the incidence was estimated; ROC: Receiver operating characteristic; PRS: Polygenic risk score(s); SD: standard deviation.

\section{Declarations}

\section{Ethics approval and consent to participate}

All participants have given informed consent and the current analysis falls under the ethics approval for the study of Läll and colleauges, granted by the Research Ethics Committee of the University of Tartu (approval 234/T-12) (27).

\section{Consent for publication}

Not applicable.

\section{Availability of data and materials}

We do not have ethical approval to share individual data for Estonian Biobank.

Access to the data set used and analysed during the current study can be requested via data application form, via https://www.geenivaramu.ee/en/access-biobank.

\section{Competing interest}

The authors have no competing interest to declare.

\section{Funding}

Funding for this project has been provided by the European Union's Horizon 2020 research and innovation programme under the Marie Skłodowska-Curie grant agreement No 676207 as well as by grant PUT1665 by the Estonian Research Council. 
$\mathrm{MO}, \mathrm{KF}, \mathrm{PMB}$, and EG developed the conceptualization of the study and chose the methodology. KF provided the EstBB data; MO performed the analysis and visualization; MO, KF, PMB and EG interpreted the analysis; MO wrote the first draft of the manuscript; PMB and EG critically reviewed and improved the first draft; all authors critically reviewed the manuscript prior to submission; PMB and EG provided supervision during of the project. The author(s) read and approved the final manuscript.

\section{Acknowledgement}

The authors thank the ECGUT for data access and Kristi Läll for introduction to the development of the PRS used in this analysis.

\section{References}

1. Ferlay J, Colombet M, Soerjomataram I, Mathers C, Parkin DM, Piñeros M, et al. Estimating the global cancer incidence and mortality in 2018: GLOBOCAN sources and methods. Int J Cancer. 2019;144:1941-53.

2. Diet, Nutrition, Physical Activity and Cancer: a Global Perspective. Continuous Update Project Expert Report 2018. World Cancer Research Fund, American Institute for Cancer Research. Available at dietandcancerreport.org. 2018.

3. International Agency for Research on Cancer, WHO. Globocan 2018. Estonia.

4. American Cancer Society. Cancer Facts and Figures 2020. Atlanta Am Cancer Soc. 2020

5. European Commission initiative on breast cancer. European Commission. Recommendations from European Breast Guidelines [Internet]. Accessed 13 Mar 2019. Available from: https://ecibc.jrc.ec.europa.eu/recommendations/

6. Nelson HD, Cantor A, Humphrey L, Fu R, Pappas M, Daeges M, et al. Screening for Breast Cancer: A Systematic Review to Update the 2009 U.S. Preventive Services Task Force Recommendation. Evid Synth. 2016;124:1-277.

7. The National Health Service (NHS). Breast cancer screening [Internet]. Accessed 16 May 2020. Available from: https://www.nhs.uk/conditions/breast-cancer-screening/

8. Marmot M, Altman DG, Cameron DA, Dewar JA, Thompson SG, Wilcox M. The benefits and harms of breast cancer screening: An independent review. Lancet. 2012;380:1778-86.

9. Advances in Breast Cancer Research - National Cancer Institute [Internet]. Accessed 9 Jul 2020. Available from: https://www.cancer.gov/types/breast/research

10. Seely JM, Alhassan T. Screening for breast cancer in 2018-what should we be doing today? Curr Oncol. 2018;25:S115-24.

11. Basu P, Ponti A, Anttila A, Ronco G, Senore C, Vale DB, et al. Status of implementation and organization of cancer screening in The European Union Member States-Summary results from the 
second European screening report. Int J Cancer. 2018;142:44-56.

12. Cancer Research UK. Breast cancer incidence (invasive) statistics [Internet]. Accessed 9 Jul 2020. Available from: https://www.cancerresearchuk.org/health-professional/cancer-statistics/statisticsby-cancer-type/breast-cancer/incidence-invasive\#heading-One

13. Integraal kankercentrum Nederland (KNL). NKR Cijfers [Internet]. Accessed 9 Jul 2020. Available from: https://iknl.nl/

14. Gøtzsche PC, Jørgensen KJ. Screening for breast cancer with mammography. Cochrane Database Syst Rev. 2013;Art. No.: CD001877.

15. Hofvind S, Ponti A, Patnick J, Ascunce N, Njor S, Broeders M, et al. False-positive results in mammographic screening for breast cancer in Europe: A literature review and survey of service screening programmes. J Med Screen. 2012;19:57-66.

16. Mavaddat N, Michailidou K, Dennis J, Lush M, Fachal L, Lee A, et al. Polygenic Risk Scores for Prediction of Breast Cancer and Breast Cancer Subtypes. Am J Hum Genet. 2019;104:21-34.

17. Torkamani A, Wineinger NE, Topol EJ. The personal and clinical utility of polygenic risk scores. Nature Reviews Genetics. 2018;19:581-90.

18. Maas P, Barrdahl M, Joshi AD, Auer PL, Gaudet MM, Milne RL, et al. Breast Cancer Risk From Modifiable and Nonmodifiable Risk Factors Among White Women in the United States. JAMA Oncol. 2016;2:1295-302.

19. Mavaddat N, Michailidou K, Dennis J, Lush M, Fachal L, Lee A, et al. Polygenic Risk Scores for Prediction of Breast Cancer and Breast Cancer Subtypes. Am J Hum Genet. 2019;104:21-34.

20. Zhang X, Rice M, Tworoger SS, Rosner BA, Eliassen AH, Tamimi RM, et al. Addition of a polygenic risk score, mammographic density, and endogenous hormones to existing breast cancer risk prediction models: A nested case-control study. PLoS Med. 2018 ;15:e1002644.

21. Shieh Y, Hu D, Ma L, Huntsman S, Gard CC, Leung JWT, et al. Breast cancer risk prediction using a clinical risk model and polygenic risk score. Breast Cancer Res Treat. 2016;159:513-25.

22. Maas P, Barrdahl M, Joshi AD, Auer PL, Gaudet MM, Milne RL, et al. Breast Cancer Risk From Modifiable and Nonmodifiable Risk Factors Among White Women in the United States. JAMA Oncol. 2016;2:1295-302.

23. Mavaddat N, P Pharoah PD, Michailidou K, Tyrer J, Brook MN, Bolla MK, et al. Prediction of Breast Cancer Risk Based on Profiling With Common Genetic Variants. JNCI J Natl Cancer Inst. 2015;107:36.

24. Vachon CM, Pankratz VS, Scott CG, Haeberle L, Ziv E, Jensen MR, et al. The Contributions of Breast Density and Common Genetic Variation to Breast Cancer Risk. JNCl J Natl Cancer Inst. 2015;107:397.

25. Mealiffe ME, Stokowski RP, Rhees BK, Prentice RL, Pettinger M, Hinds DA. Assessment of Clinical Validity of a Breast Cancer Risk Model Combining Genetic and Clinical Information. JNCI J Natl Cancer Inst. 2010;102:1618-27. 
26. Wacholder S, Hartge P, Prentice R, Garcia-Closas M, Feigelson HS, Diver WR, et al. Performance of common genetic variants in breast-cancer risk models. N Engl J Med. 2010;362:986-93.

27. Läll K, Lepamets M, Palover M, Esko T, Metspalu A, Tõnisson N, et al. Polygenic prediction of breast cancer: comparison of genetic predictors and implications for risk stratification. BMC Cancer. 2019;19:557.

28. Steyerberg EW, Vickers AJ, Cook NR, Gerds T, Gonen M, Obuchowski N, et al. Assessing the performance of prediction models: A framework for traditional and novel measures. Epidemiology. 2010;21:128-38.

29. Leitsalu L, Haller T, Esko T, Tammesoo M-L, Alavere H, Snieder H, et al. Cohort Profile: Estonian Biobank of the Estonian Genome Center, University of Tartu. International Journal of Epidemiology. 2015;44:1137-47.

30. Estonian Genome Center, 2001-2011. Est Genome Center, Univ Tartu. 2011. Available from: https://genomics.ut.ee/sites/default/files/geenivaramu/estoniangenomecenter.pdf

31. University of Tartu Institute of Genomics [Internet]. Accessed 9 Jul 2019. Available from: https://www.geenivaramu.ee/en/about-us

32. General information - Statistics Estonia [Internet]. Accessed 9 Jul 2020. Available from: https://www.stat.ee/26262

33. Milani L, Leitsalu L, Metspalu A. An epidemiological perspective of personalized medicine: the Estonian experience. J Intern Med. 2015;277:188-200.

34. Innos K, Valvere V, Padrik P, Eelma E, Kütner R, Lehtsaar J, et al. Mammography use and mode of detection among breast cancer patients in Estonia. Women Heal. 2016;56:129-40.

35. Pencina MJ, D'Agostino RB, D’Agostino RB, Vasan RS. Evaluating the added predictive ability of a new marker: From area under the ROC curve to reclassification and beyond. Stat Med. 2008;27:15772.

36. Läll K, Mägi R, Morris A, Metspalu A, Fischer K. Personalized risk prediction for type 2 diabetes: The potential of genetic risk scores. Genet Med. 2017;19:322-9.

37. Hüsing A, Canzian F, Beckmann L, Garcia-Closas M, Diver WR, Thun MJ, et al. Prediction of breast cancer risk by genetic risk factors, overall and by hormone receptor status. J Med Genet. 2012;49:601-8.

38. Darabi H, Czene K, Zhao W, Liu J, Hall P, Humphreys K. Breast cancer risk prediction and individualised screening based on common genetic variation and breast density measurement. Breast Cancer Res. 2012;14:R25.

39. Pepe MS, Janes H, Longton G, Leisenring W, Newcomb P. Limitations of the Odds Ratio in Gauging the Performance of a Diagnostic, Prognostic, or Screening Marker. Am J Epidemiol. 2004;159:88290.

40. Ware JH. The Limitations of Risk Factors as Prognostic Tools. N Engl J Med. 2006;355:2615-7. 
41. Wald NJ, Hackshaw AK, Frost CD. When can a risk factor be used as a worthwhile screening test? BMJ. 1999;319:1562.

42. Pepe MS, Janes H, Longton G, Leisenring W, Newcomb P. Limitations of the Odds Ratio in Gauging the Performance of a Diagnostic, Prognostic, or Screening Marker. Am J Epidemiol Hopkins Bloom Sch Public Heal All rights Reserv. 2004;159:882-90.

43. Estonian Genome Centre, University of Tartu Institute of Genomics [Internet]. Accessed 20 Oct 2020. Available from: https://genomics.ut.ee/en/about-us/estonian-genome-centre

44. BBMRI.ee: Estonian Biobank has now recruited over 200,000 biobank participants and all will be genotyped by June 2020. University of Tartu Institute of Genomics [Internet]. Accessed 20 Oct 2020. Available from: https://genomics.ut.ee/en/news/bbmriee-estonian-biobank-has-now-recruited-over200000-biobank-participants-and-all-will-be

45. Estonia Houses The Biggest Biobank In Europe [Internet]. Accessed 20 Oct 2020. Available from: https://www.forbes.com/sites/yiannismouratidis/2019/01/30/estonia-houses-the-biggest-biobankin-europe/\#187a6658227a

46. Home Page - MyPeBS [Internet]. Accessed Jul 9 2020. Available from: https://mypebs.eu/

\section{Table}

Table 4 not available with this version

\section{Figures}

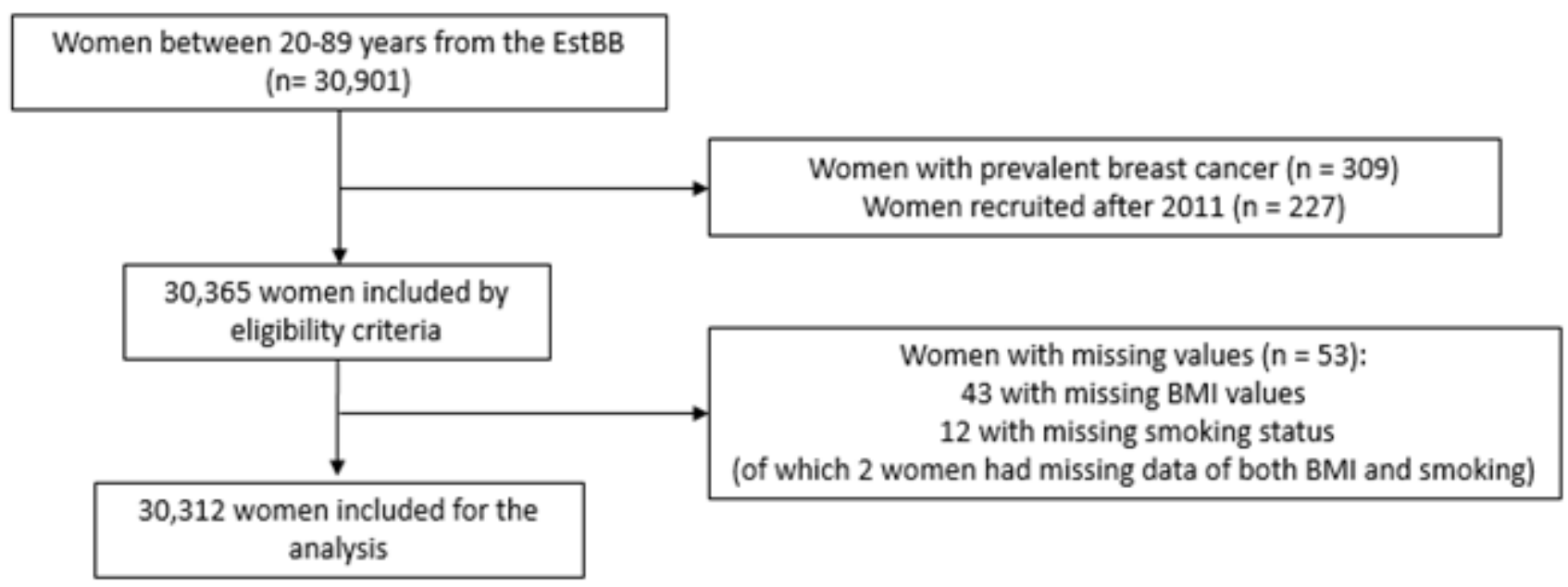

\section{Figure 1}

Flow diagram of participants included in the analysis 


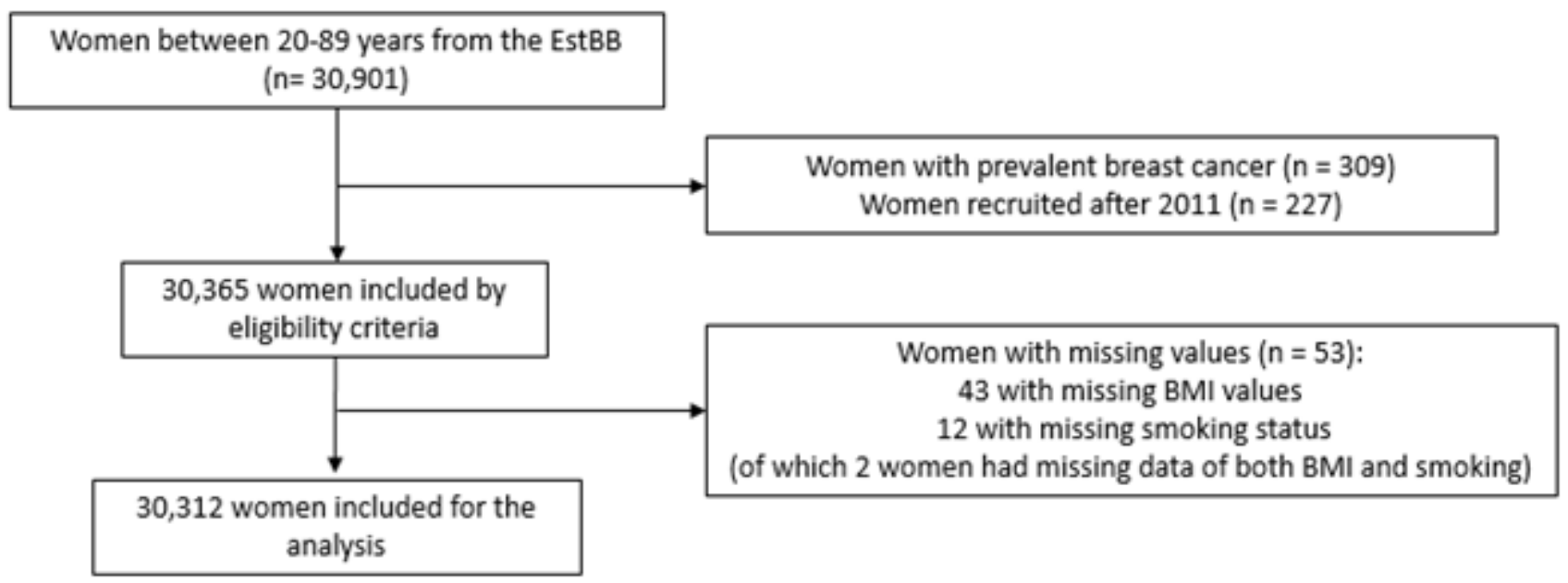

\section{Figure 1}

Flow diagram of participants included in the analysis 
a) Breast cancer before death

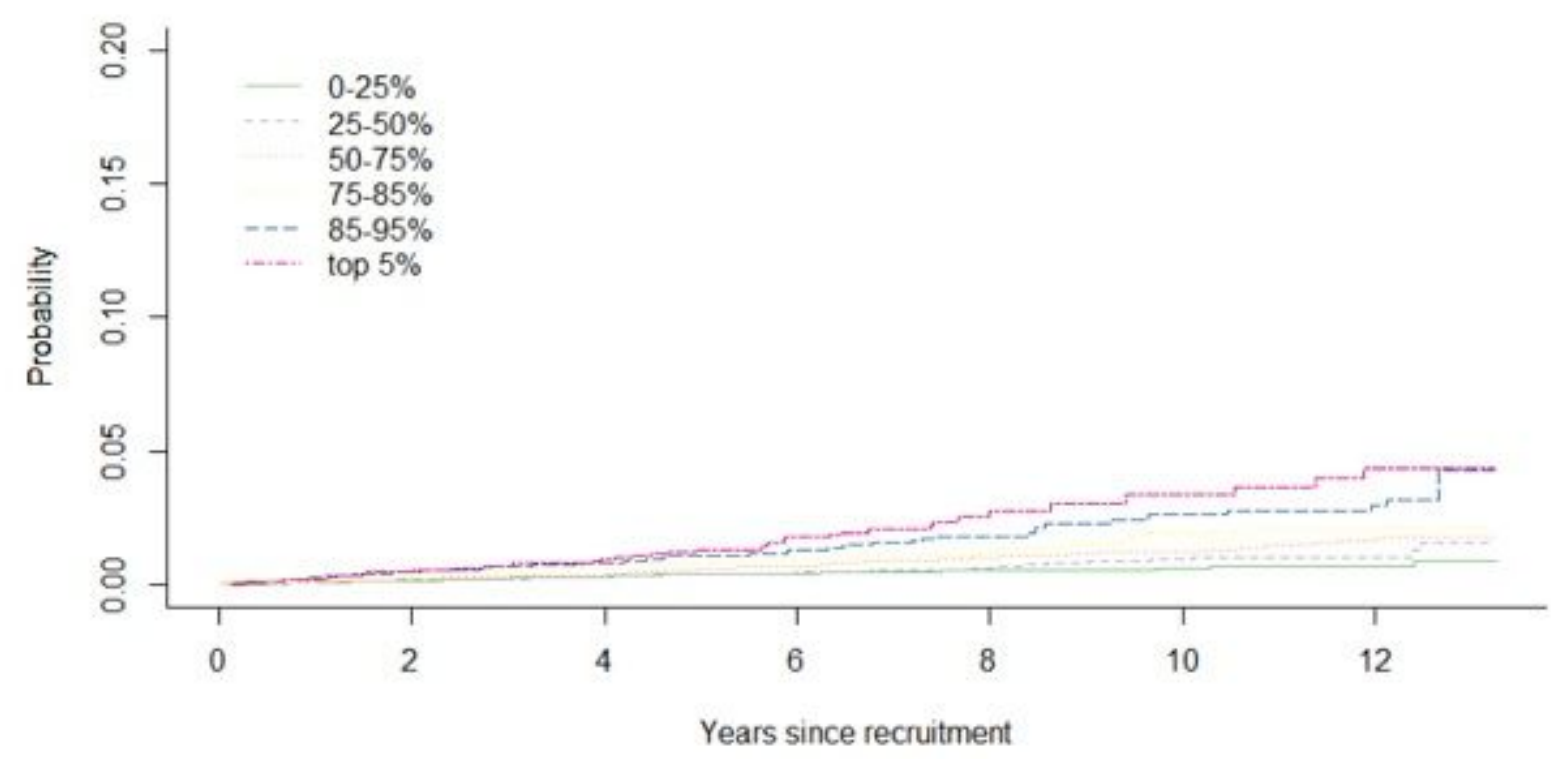

d) Death without breast cancer

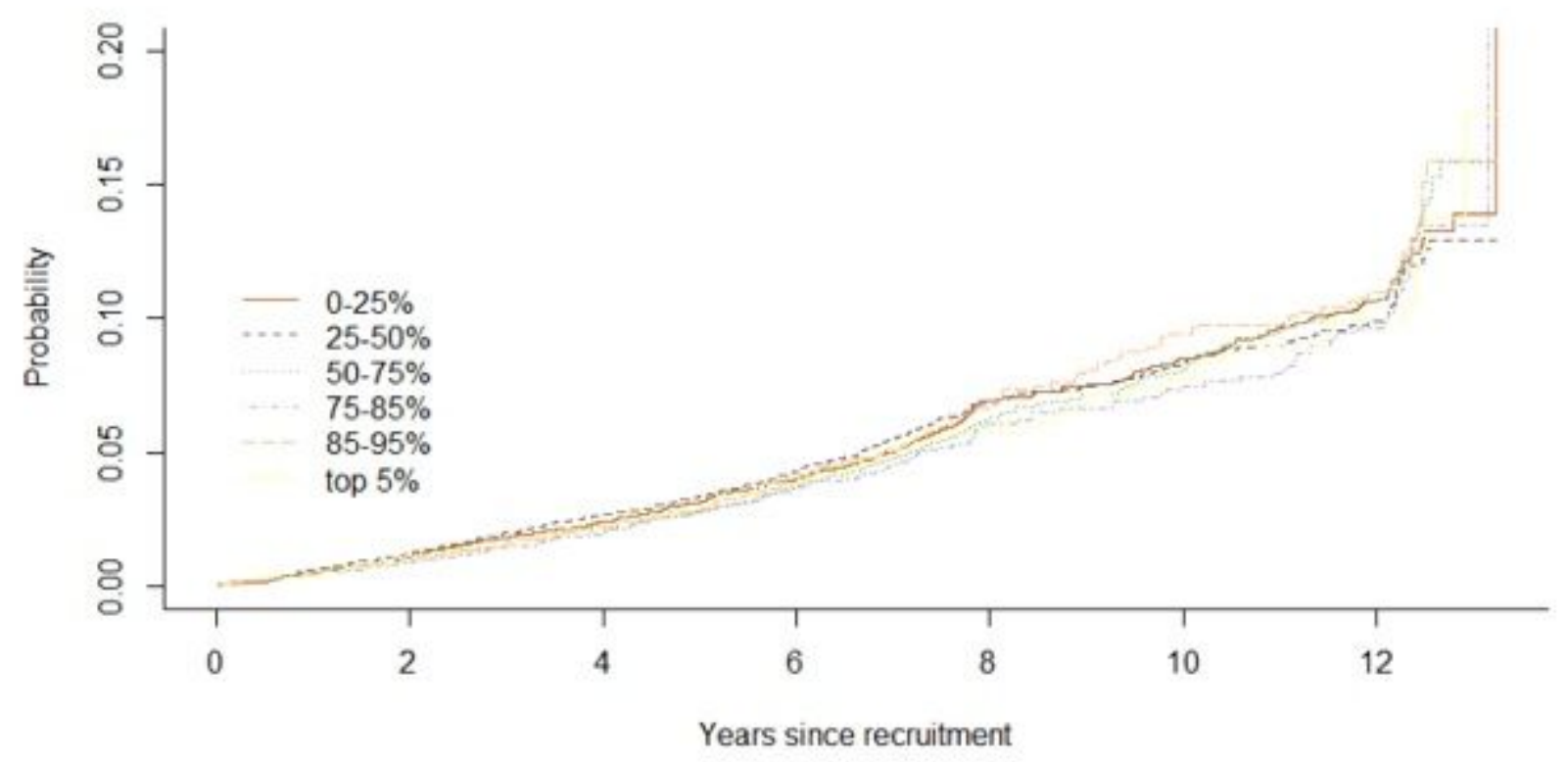

Figure 2

Unadjusted cumulative incidence curves stratified by PRS groups Legend: The time scale is years since recruitment until time of last follow-up of breast cancer 
a) Breast cancer before death

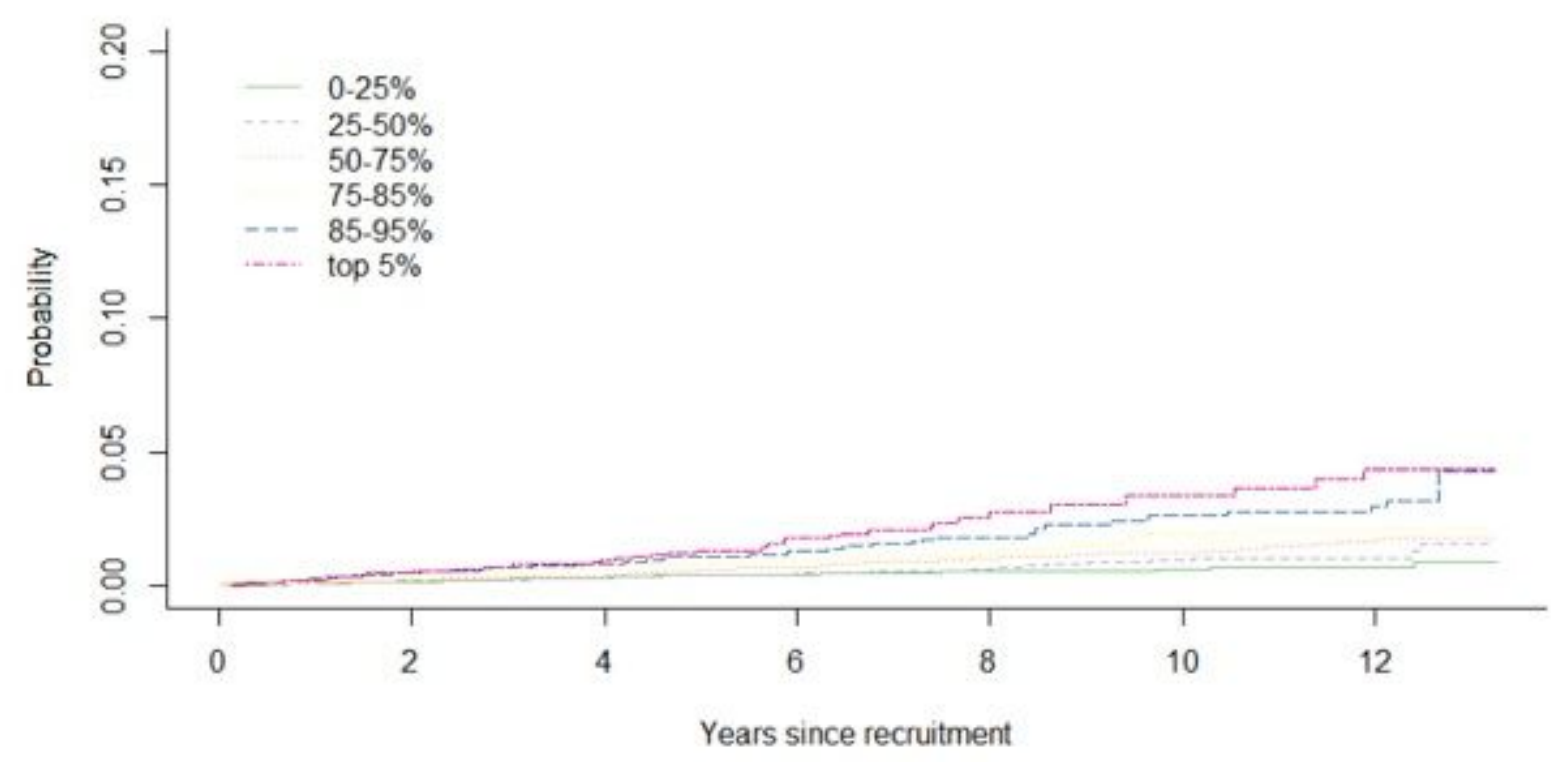

d) Death without breast cancer

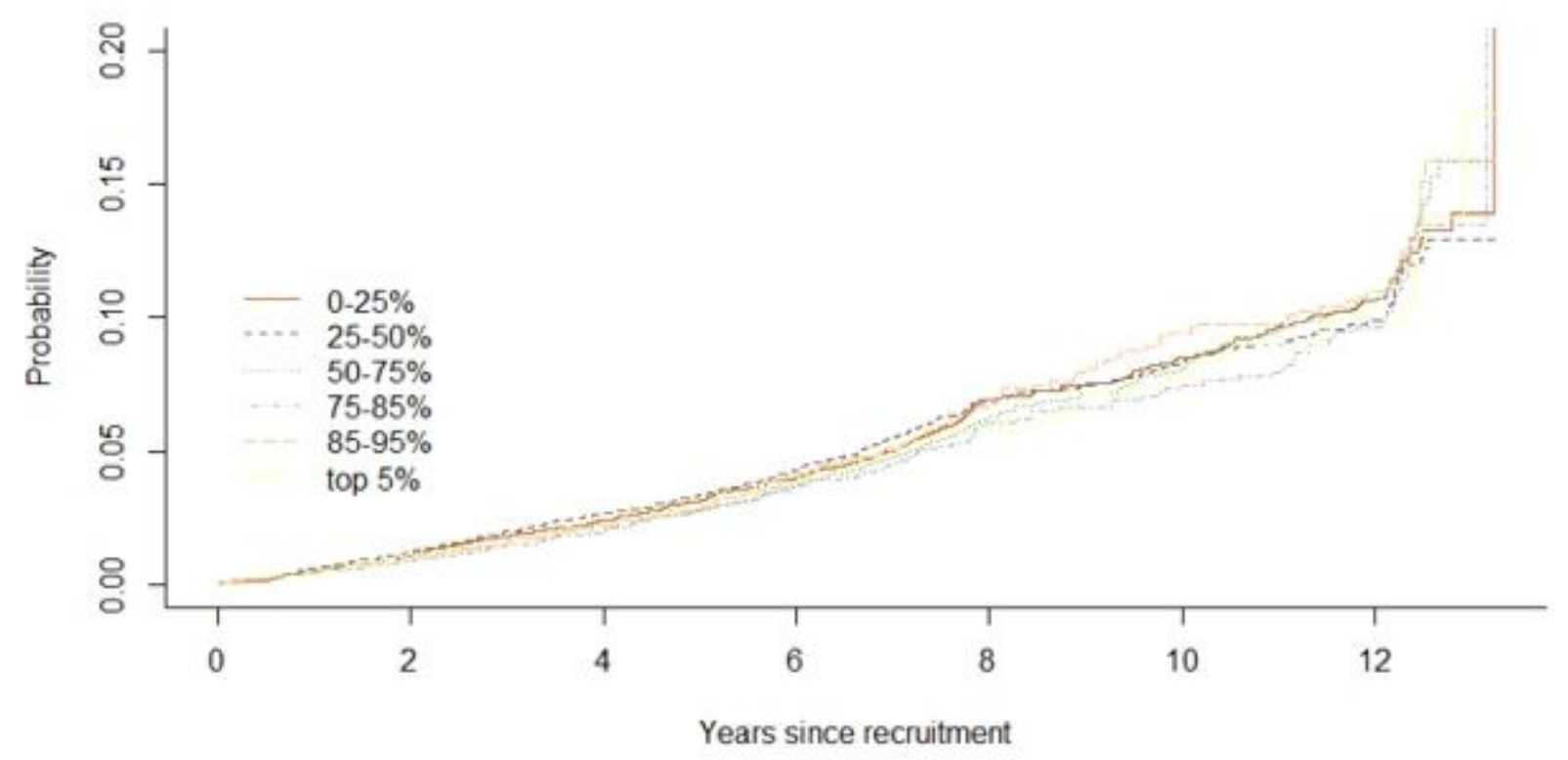

Figure 2

Unadjusted cumulative incidence curves stratified by PRS groups Legend: The time scale is years since recruitment until time of last follow-up of breast cancer 
a) 3-year incidences

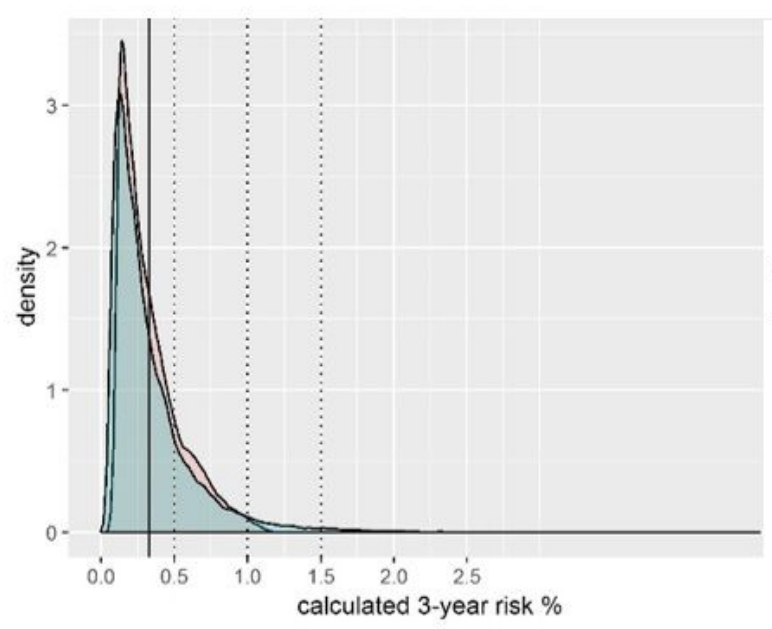

b) 5-year incidences

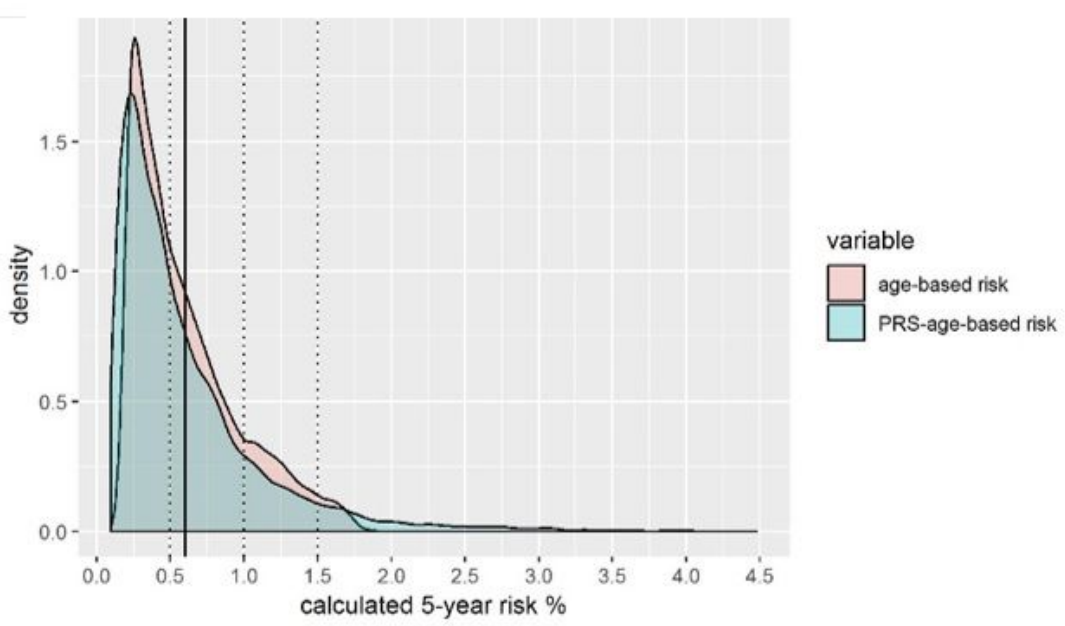

Figure 3

Three- and 5-year PRSage_mlnc and PRSage_mlnc distributions Legend: The figure shows the absolute incidence distributions for the age_mInc (red) and PRSage_mInc (blue). The full vertical line shows the mean PRSage_mlnc and the dotted lines the $0.5 \%, 1 \%$ and the $1.5 \%$ risk thresholds, respectively.

a) 3-year incidences

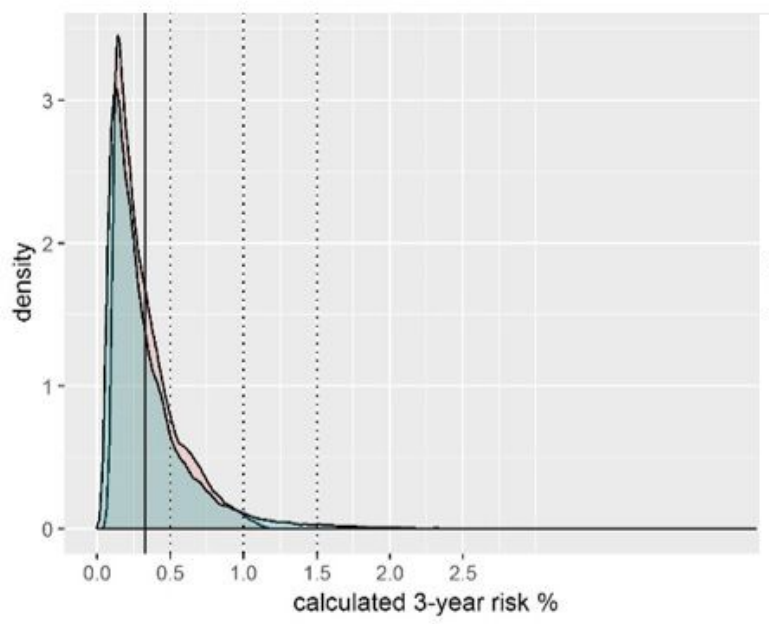

b) 5-year incidences

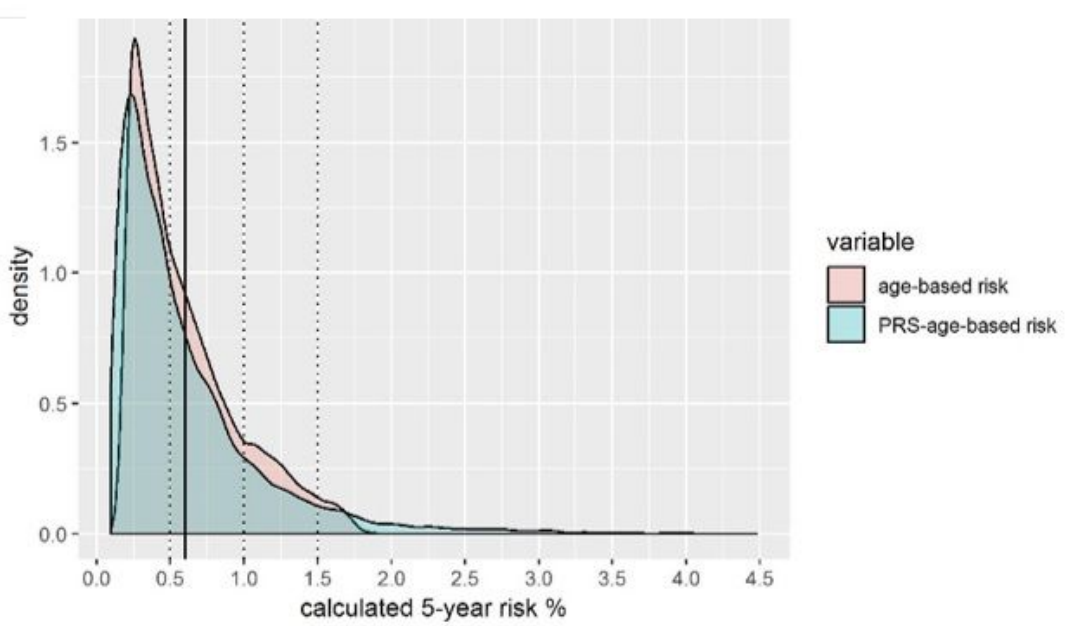

\section{Figure 3}

Three- and 5-year PRSage_mInc and PRSage_mlnc distributions Legend: The figure shows the absolute incidence distributions for the age_mInc (red) and PRSage_mlnc (blue). The full vertical line shows the mean PRSage_mlnc and the dotted lines the $0.5 \%, 1 \%$ and the $1.5 \%$ risk thresholds, respectively. 
a) 3-year incidences

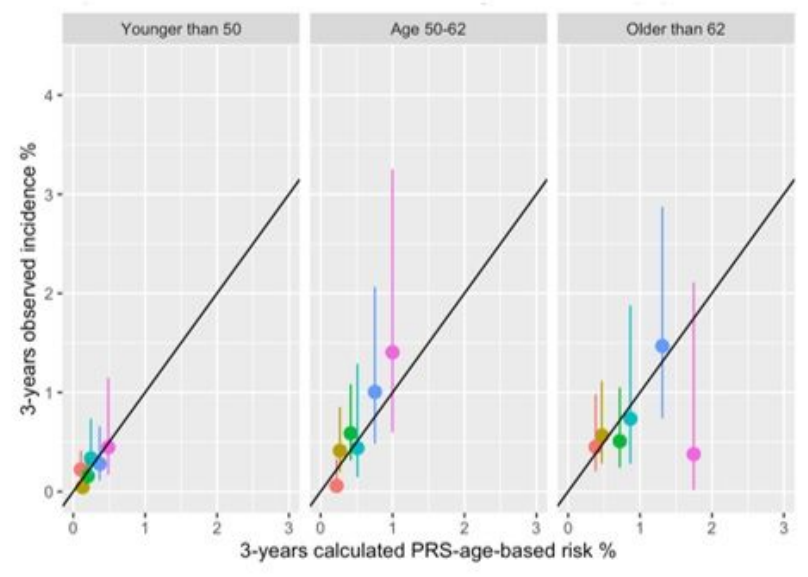

b) 5-year incidences

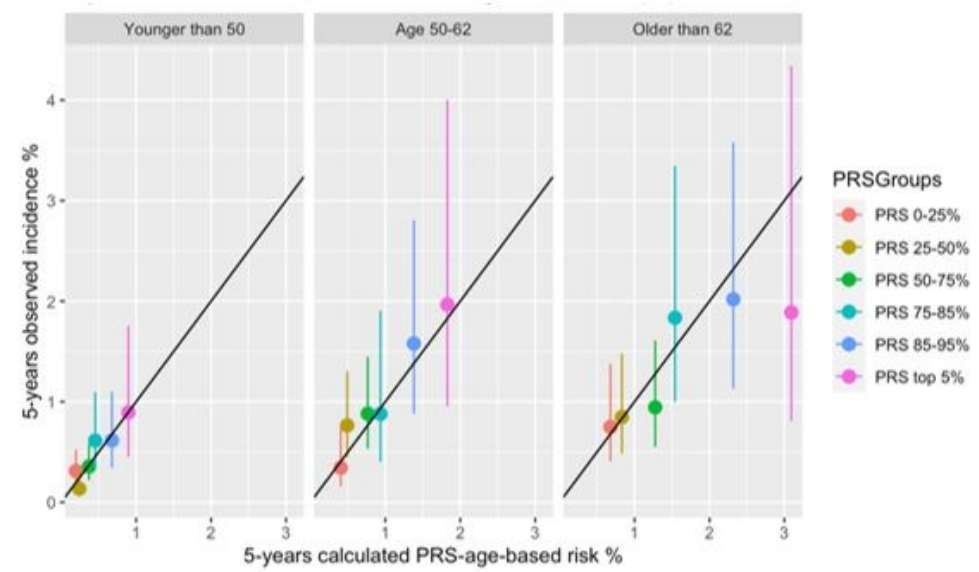

Figure 4

Three- and 5-year incidence calibration plots Legend: The figure shows calibration plots of the observed cumulative incidence (incl. 95\% Cl) plotted on the y-axis against the mean PRSage_mlnc, for each PRSage subgroup.

a) 3-year incidences

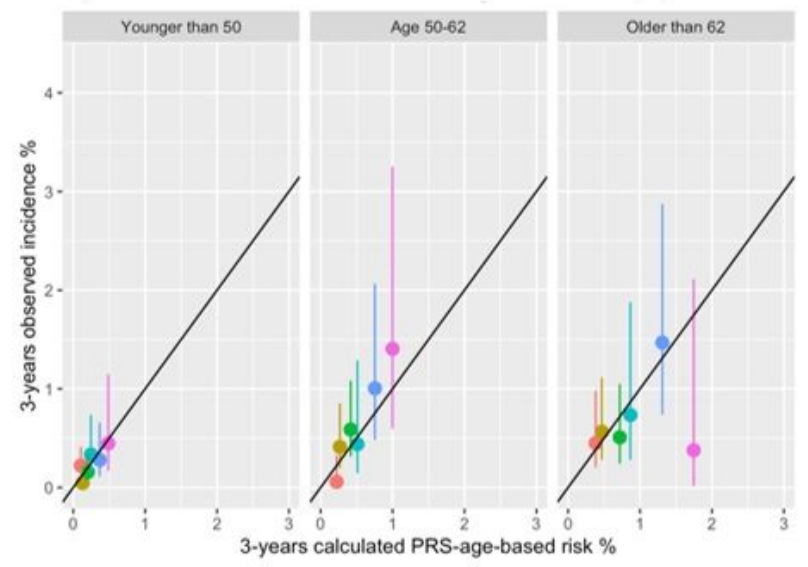

b) 5-year incidences

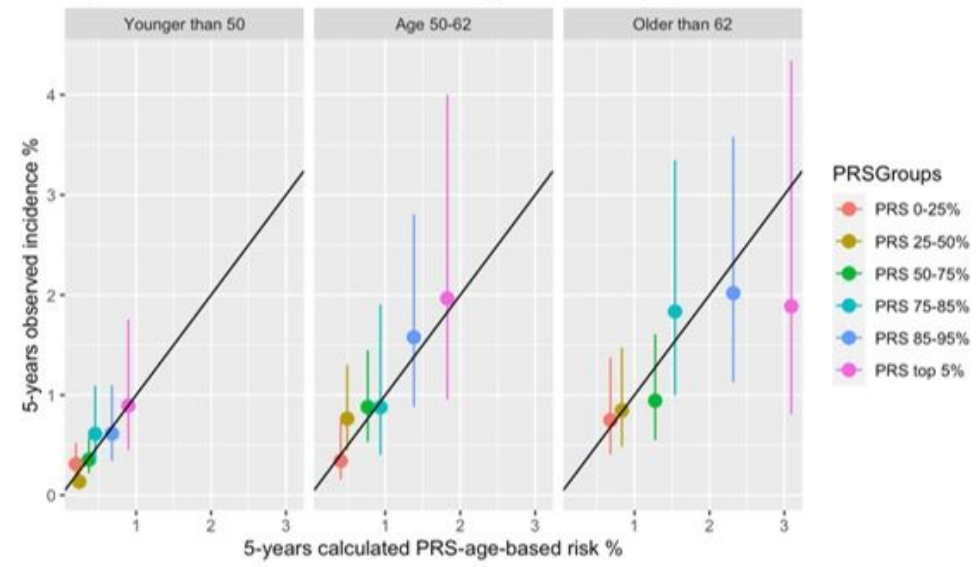

\section{Figure 4}

Three- and 5-year incidence calibration plots Legend: The figure shows calibration plots of the observed cumulative incidence (incl. $95 \% \mathrm{Cl}$ ) plotted on the $y$-axis against the mean PRSage_mlnc, for each PRS- 
age subgroup.

a) 3-years PRSage_mInc

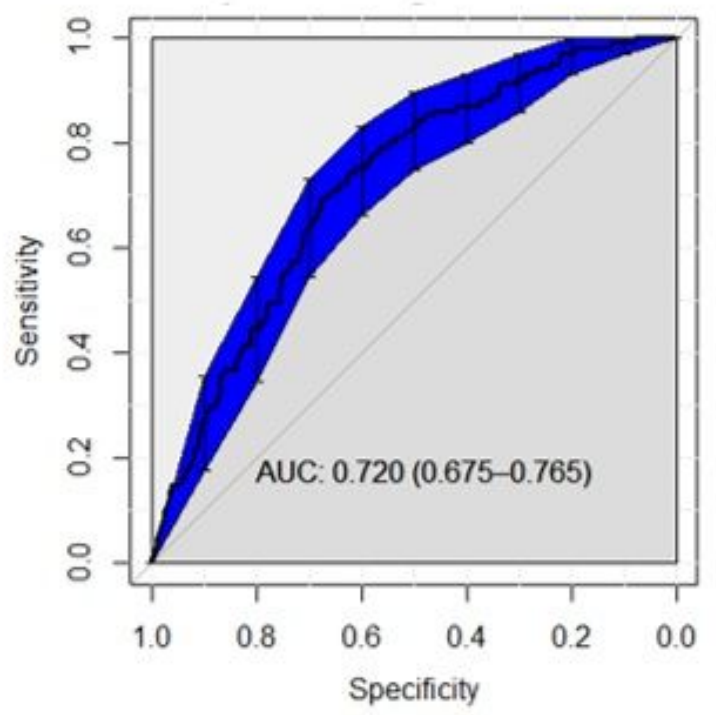

c) 5-years PRSage_mInc

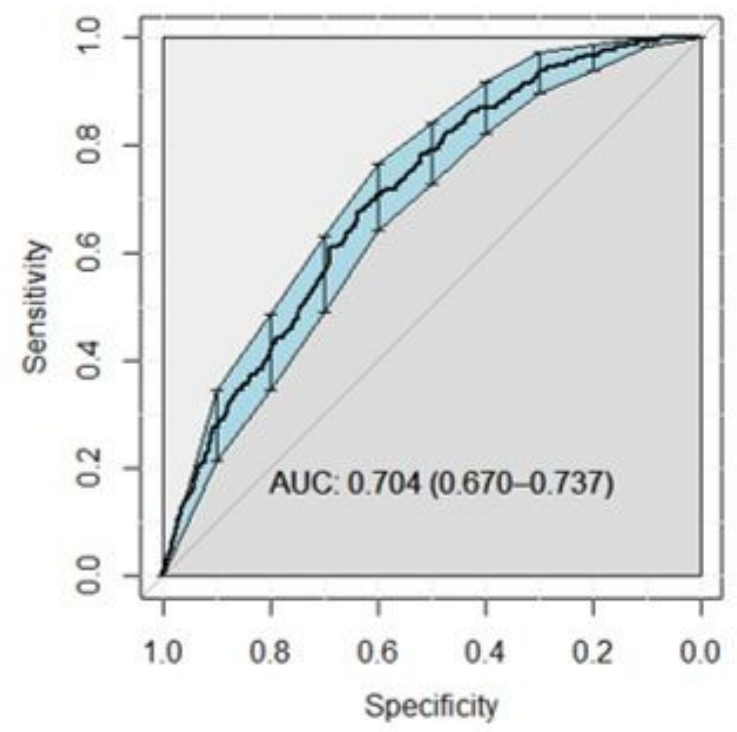

b) 3-years age mInc

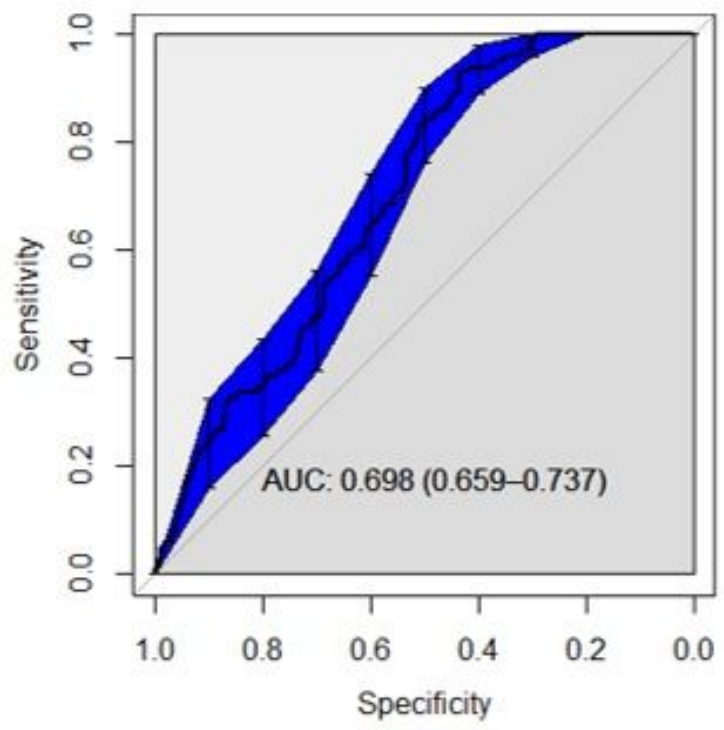

d) 5-years age_mInc

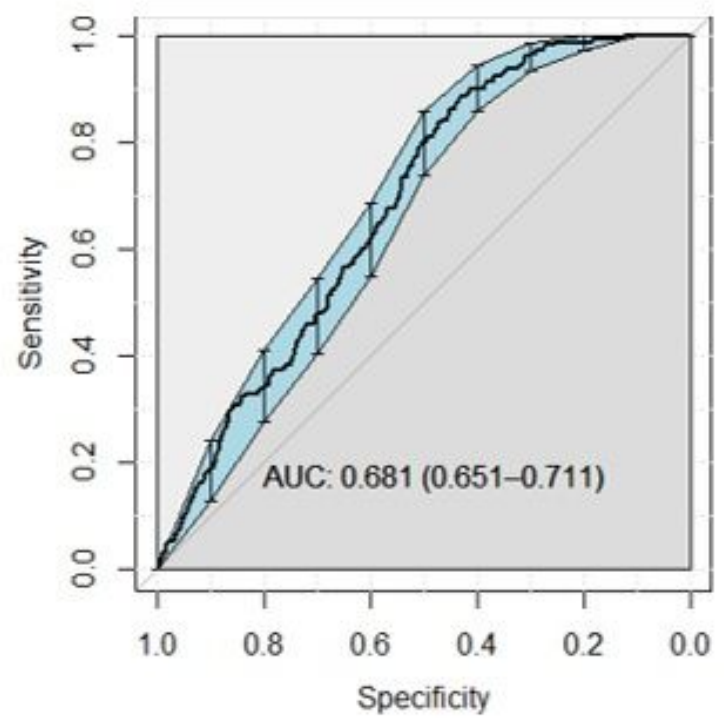

Figure 5

ROC and corresponding AUC of the PRSage_mlnc and age_mlnc Legend: The figure shows the ROC curve and AUC of the PRSage_mlnc and age_mlnc. 
a) 3-years PRSage_mInc

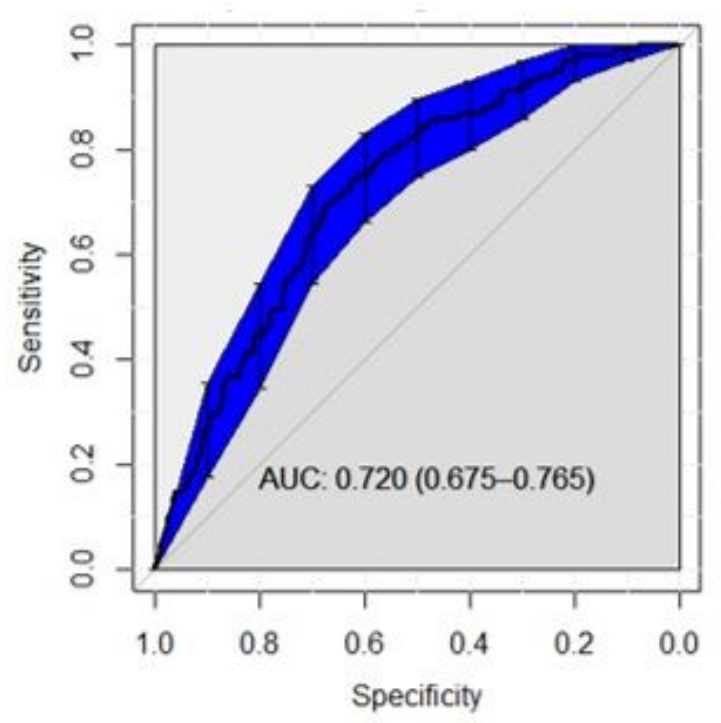

c) 5-years PRSage_mInc

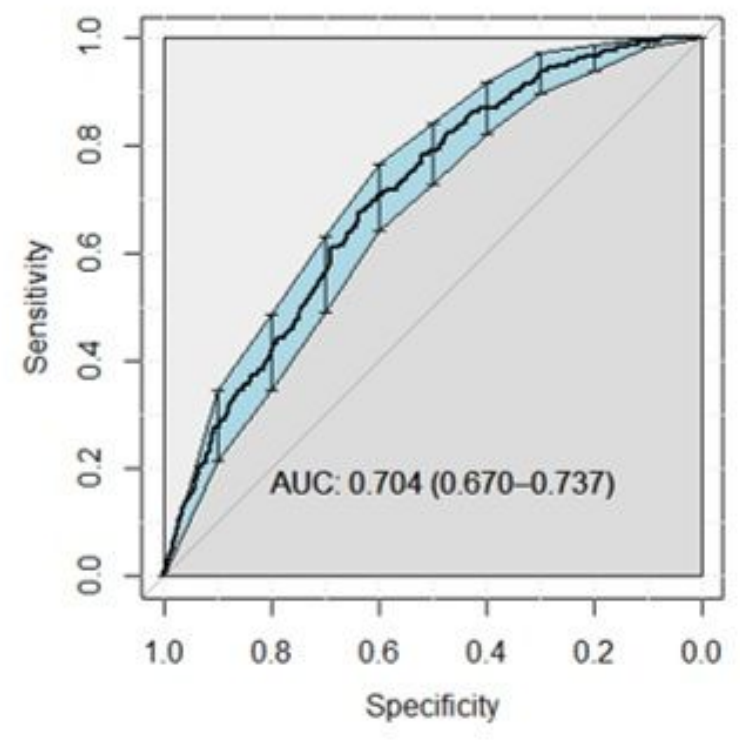

b) 3-years age_mInc

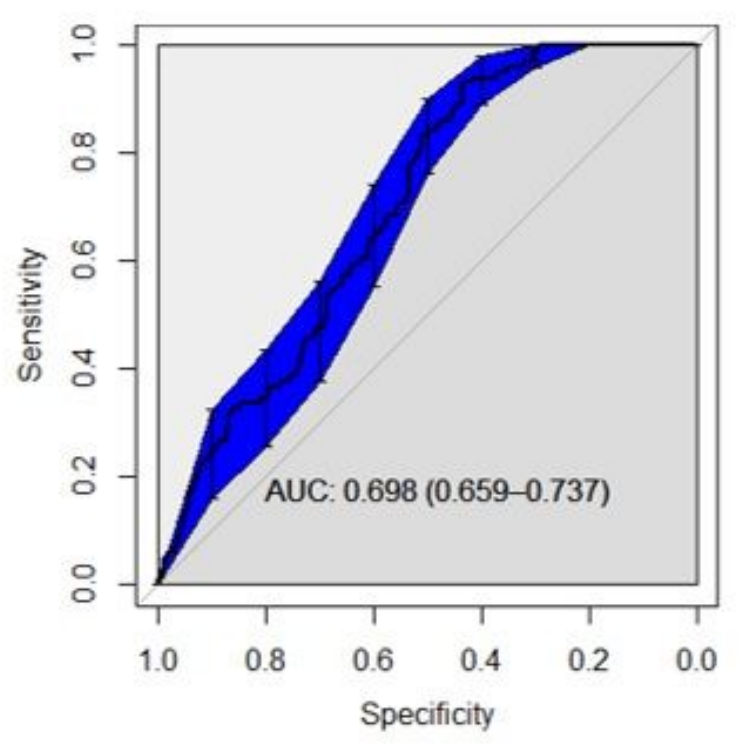

d) 5-years age_mInc

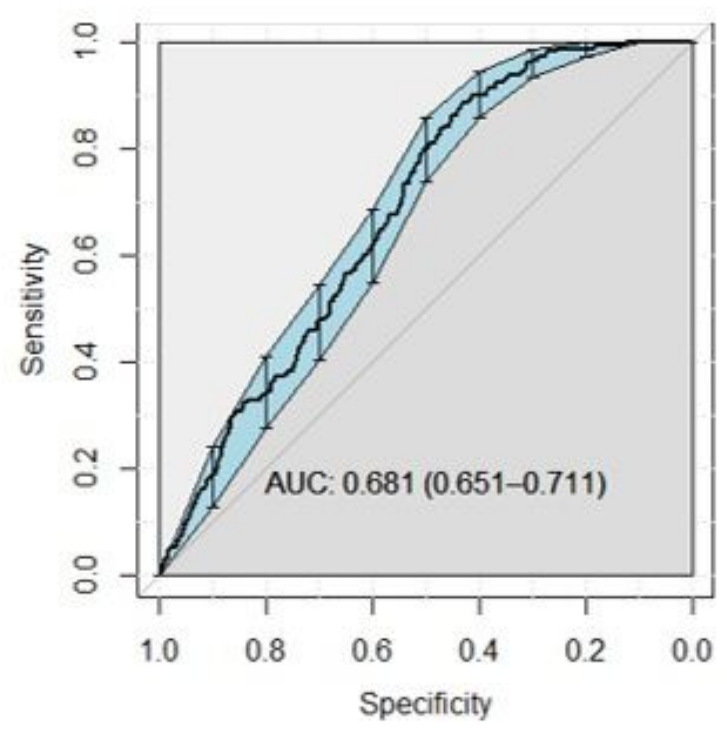

\section{Figure 5}

ROC and corresponding AUC of the PRSage_mlnc and age_mlnc Legend: The figure shows the ROC curve and AUC of the PRSage_mlnc and age_mlnc.

\section{Supplementary Files}

This is a list of supplementary files associated with this preprint. Click to download.

- Supplementaryfile05.11.20.docx 
- Supplementaryfile05.11.20.docx

Page $30 / 30$ 